\title{
70 years of Landau-Teller theory for collisional energy transfer. Semiclassical three-dimensional generalizations of the classical collinear model
}

\author{
E. E. Nikitin ${ }^{a b}$ and J. Troe ${ }^{* b c}$ \\ Received 1st October 2007, Accepted 7th November 2007 \\ First published as an Advance Article on the web 28th November 2007 \\ DOI: $10.1039 / \mathbf{b} 715095 d$
}

This article, in historical retrospective, describes the development of the celebrated Landau-Teller (LT) model of 1936 for vibrational-translational energy exchange in collisions of an atom with a diatomic molecule. We discuss semiclassical generalizations of the classical LT model and generalizations of the collinear LT model to account for the effects of rotation of the diatom on the vibrational relaxation rate. The former is based on the recovery of the Landau semiclassical exponent from the classical LT encounter time, and the latter on the definition of a 1-D driving mode within the manifold of the translational and rotational degrees of freedom of the colliding partners. The utility of generalized LT models is illustrated by three case studies that exemplify weak and strong effects of the rotation as well as the efficiencies of different driving modes in the vibrational relaxation of highly asymmetric diatoms.

\section{Introduction}

More than 70 years ago, Landau and Teller published their article on the mechanism of energy transfer in molecular collisions. ${ }^{1}$ Their work had enormous impact on the understanding of this important process and it continues to do so until today. In detail, Landau and Teller (LT) explained the reasons for the low rate of the vibrational-translational energy exchange in diatomic gases which, among other effects, man-

\footnotetext{
${ }^{a}$ Schulich Faculty of Chemistry, Technion-Israel Institute of Technology, Haifa, 32000, Israel

${ }^{b}$ Max-Planck-Institut für Biophysikalische Chemie, Am Fassberg, D-37077 Göttingen, Germany

${ }^{c}$ Institut für Physikalische Chemie der Universität Göttingen,

Tammannstrasse 6, D-37077 Göttingen, Germany
}

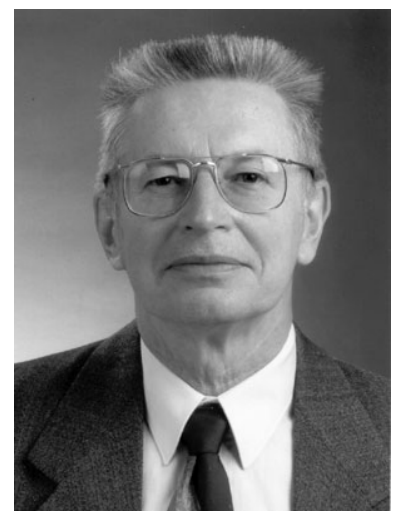

E. E. Nikitin was born in Saratov, USSR, in 1933. In 1955 graduated from the Saratov University. Since 1958 he has conducted a post-graduate study, and obtained PhD and Doctor degree in Physics, Professor of Chemical Physics and Head of the Research Group at the Institute of Chemical Physics, Academy of Sciences, Moscow. Since 1992, he has been Professor of Physical Chemistry at the Technion, Haifa, Israel, and a guest professor at Göttingen University and at the Max-Planck Institute for Biophysical Chemistry at Göttingen. Member of Academies, notably the Leopoldina and the International Academy of Quantum Molecular Sciences. Author of 15 books and about 300 articles. ifests itself in ultrasonic dispersion. They determined the temperature dependence of vibrational state-to-state rate coefficients, set up kinetic equations for the population of molecular states, and derived the relaxation equation for the average vibrational energy of the molecular ensemble. These results were considered to be of such large importance that the article was cited before it was published. ${ }^{2}$ Years later it was realized that the LT results characterize non-equilibrium phenomena in gas dynamics in general, e.g. in the transient state of a gas behind shock waves. With increasing interest in this problem during the World War II, the LT relaxation equation, now dubbed the Bethe-Teller equation, became even part of classified documents. ${ }^{3}$

The LT results referred to collinear collisions. As a consequence there was no way to estimate the absolute value of the

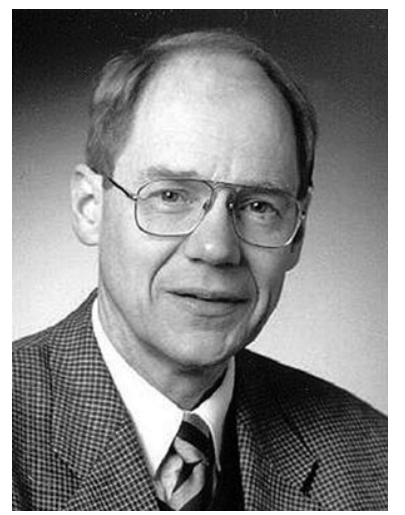

J. Troe was born in Göttingen, Germany, in 1940. In 1965 he obtained $a \mathrm{PhD}$ and in 1968 a habilitation degree in physical chemistry from Göttingen University. In the period of 1971-1975 he was a Professor of Physical Chemistry at the Ecole Polytechnique Fédérale de Lausanne, Switzerland. Since 1975 J. Troe was active as Professor of Physical Chemistry at Göttingen University and since 1990 also as Director at the Max-Planck Institute for Biophysical Chemistry at Göttingen. He obtained honorary doctorates from the Universities of Bordeaux and Karlsruhe. Member of Göttingen and Berlin-Brandenburg Academy, Leopoldina, Academia Europaea and the American Academy of Arts and Sciences. He is author of about 450 articles. 
rate coefficients. Besides, the model was restricted to situations when the vibrational quantum $\hbar \omega$ of the harmonic molecular oscillator was much smaller than $k_{\mathrm{B}} T$ (hence called here the "classical model"). The first attempt to relax the condition $\hbar \omega / k_{\mathrm{B}} T \ll 1$ goes back to Schwartz, Slawsky, and Herzfeld ${ }^{4}$ who resorted to the quantum solution of the collinear collision model by Jackson and Mott, ${ }^{5}$ the classical counterpart of which was also used by Landau and Teller. ${ }^{1}$ Three-dimensional versions of this model were proposed by Takayanagi ${ }^{6}$ and Schwartz and Herzfeld. ${ }^{7}$ The ultimate result of ref. 4 and 7, known as the SSH theory, for the next decade became a cornerstone of the interpretation of various vibrational nonequilibrium phenomena in gases. ${ }^{8}$

SSH theory used the model of a three-dimensional collision with a short-range isotropic repulsion between a vibrating molecule and a structureless particle, hence it was also called the breathing sphere (BS) model. In an attempt to account for the effect of the vibrational anisotropy of the diatom, it was recommended that the $\mathrm{BS}$ rate coefficient be multiplied by a steric factor of about $1 / 3 .^{7}$ The $\mathrm{SSH}$ treatment was suggestive of an application of a regular perturbation approach with respect to the anisotropic part of the interaction. The anticipated effect of the anisotropy on the vibrational relaxation rate stemmed from considerations that an anisotropic interaction should induce simultaneous vibrational and rotational transitions, thus diminishing the amount of energy liberated in the form of translation. Unfortunately, the approach within the vibrational-rotational perturbation theory of the lowestorder was not successful since one had to resort to a basis for which the rotational-translational coupling of the atom-nonvibrating diatom system was fully accounted for. Such a basis cannot be found in general. However, it can be determined in the particular case when the collision is considered as a sudden event with respect to the rotational period of the molecule, and when the Coriolis coupling in the body-fixed frame of the colliding partners is ignored. Formally, this is done by summation of an infinite perturbation series in the free rotor basis, hence the acronym IOS for "infinite order sudden". (The IOS as well as CS, "coupled states", and CC, "close-coupling", nomenclatures are standard ones in the theory of molecular collisions. We therefore refer to them as they are described in the numerous textbooks, e.g. ref. 9-13). The result of the IOS approximation is a rather simple generalization of the BS model, within which the collision occurs in an effective isotropic potential which parametrically depends on the conserved orientation angle of the molecular axis with respect to the collision axis. The rate coefficient is obtained by the averaging over the orientation angles. (We note in passing that the IOS approximation differs from the standard sudden approximation in that the latter assumes a fixed orientation of the molecular axis in a spaced-fixed frame rather than in the body-fixed (BF) frame.)

The IOS approximation partially takes into account the anisotropy of interaction but it totally ignores effects related to channelling part of the released vibrational energy into the rotational degrees of freedom of the diatom. A considerably better approximation is obtained when, still ignoring the Coriolis interaction in the $\mathrm{BF}$ frame, the assumption of a sudden nature of the collision with respect to rotational transitions is dropped. Within this approach, i.e. the coupled state (CS) approximation, the projection of the intrinsic angular momentum onto the collision axis is assumed to be a conserved quantity. This partially restricts the coupling between different rotational states. The rate coefficient within the CS approximation is obtained by the averaging over all possible projections of the intrinsic angular momenta.

The CS approximation differs from the accurate approach, conventionally called the close-coupling (CC) method, only in that it neglects the Coriolis interaction in the $\mathrm{BF}$ frame. Once the Coriolis coupling is finally also taken into account, as this is the case within the $\mathrm{CC}$ method, the projection of the intrinsic angular momentum onto the collision axis is not conserved anymore. Nonetheless, vibrational relaxation rate coefficients calculated by CS and CC methods were found to be close to each other. This can be understood as the result of an averaging over different projections in a well-localized region of the potential where the vibrational transition takes place. One should note that the latter feature of the vibrational coupling was a basic property already in the original LT model.

The hierarchy of approaches of ever-increasing complexity, $\mathrm{LT} \rightarrow \mathrm{SSH}(\mathrm{BS}) \rightarrow \mathrm{IOS} \rightarrow \mathrm{CS} \rightarrow \mathrm{CC}$, illustrates the relation between the original classical collinear Landau-Teller model with exponential repulsive interaction and the full quantum treatments of the 3-D collision dynamics on ab initio potential energy surfaces. The nowadays available numerical codes make CS and CC calculations of vibrational state-to-state rate coefficients an easy task for simple inelastic processes such as collisions of closed-shell atoms with diatomic molecules in nondegenerate electronic states. Our interest in the Landau-Teller model stems from the observation that in the majority of practical cases the collisions occur in the "quasiclassical" (in the sense of the WKB approximation) and nearadiabatic (with respect to the vibrational transitions) regimes which are disguised in quantum scattering computations. The question addressed in the present article is whether these two conditions can be used for generalization of the original LT model, with the aim to formulate useful analytical models of SSH type which are applicable to arbitrary interaction potentials and which account for the contribution of rotations to the vibrational relaxation. In answering this question, in line with the original LT approach, we restrict ourselves to the determination of the temperature dependence of the rate coefficients rather than to the full calculation of the rates. We employ the semiclassical near-adiabatic Landau method, ${ }^{14}$ the recovery of the Landau exponent in the expression for the transition probability from the classical Landau-Teller time, ${ }^{15,16}$ and generalizations of the original LT idea that a single mode of the heat bath induces the vibrational transition. We also restrict ourselves to direct collisions when the vibrational relaxation through a transient complex as a result of the translational-rotational energy exchange can be discarded.

This objective sets the scene for discussing, in a review style representation, semiclassical three-dimensional generalizations of the classical collinear Landau-Teller model. Accordingly, the plan of the article is the following. Section 2 shortly describes the historical situation before the publication of the Landau-Teller article. Section 3 presents the main results of 
the LT approach. Section 4 provides a semiclassical generalization of the classical collinear LT model. Section 5 is devoted to two different 3-D generalizations of the collinear model, i.e. the effective mass and the hindered rotor models. Section 6 , by way of case studies, illustrates various aspects of the generalized LT models. In summary, the present review commemorates Landau and Teller's seminal work at its 70th anniversary and after its generalizations it exemplifies the usefulness of this approach until today.

\section{Approaches before Landau and Teller's work}

Inspired by the observation of many rebounds of a steel ball from a rigid steel plate, Jeans ${ }^{17}$ posed the question about molecular elasticity that would permit an exchange of translational and vibrational energy in a molecular gas. He simulated an inelastic atom-molecule collision by an external bellshaped force acting on the molecular oscillator, calculated the energy change of the molecule and compared it to the initial translational energy. In this way, a rough estimate for the vibration-translation (VT) transition probability was obtained for two types of forces, Lorentzian and Gaussian. However, due to uncertain values of the parameters substituted into the final expression for the energy change, the conclusion reached was only qualitative: the molecular "elasticity" is very high, and the VT transition probability is very low. In this work, based totally on a classical picture, no attempt was made to relate the perturbing force to the intermolecular potential.

Quite some time later, Zener ${ }^{18}$ used a semiclassical perturbative approach to calculate the VT transition probability, $P$, for an exponential repulsive potential between molecular oscillators. This method, which is today known as the common trajectory approximation, or the Ehrenfest mean field approximation or yet the external field approximation, yielded a 'reasonable' value of $P$ of about $10^{-5}$ for the deactivation of the first vibrational state (for an energy level spacing of 0.02 $\mathrm{eV}$ and collisions at room temperature). In this work, the classical perturbing force was related to the intermolecular potential and limitations for a common trajectory approximation (not too low collision energies) were formulated. These limitations were removed a year later by Jackson and Mott ${ }^{5}$ who, within the distorted wave approximation, considered the quantum problem of atom-diatom collinear collisions with a repulsive exponential potential. A consistent quantum picture of inelastic collisions was presented which made it possible to consider collisions at low energies as well as to derive an analytical expression for the VT transition probability. A more general quantum model was investigated in a series of papers by Lennard-Jones et al. ${ }^{19-21}$ in which the authors used a Morse potential. A quantum picture of inelastic events was presented for free-free, bound-free and bound-bound events. In the light of these approaches everything seemed well prepared for approximate calculations of VT rate coefficients, at least for simple models. Why then was the article by Landau and Teller considered to be of such importance that its main results were cited already in $1935 ?^{2}$ The answer to this question was obviously the fact that Landau and Teller succeeded to derive a simple expression for the temperature dependence of the state-specific vibrational rate coefficients and, at the same time, to set up the relaxation equation for the mean vibrational energy by expressing the relaxation time through the vibrational rate of deactivation of the first vibrational level of the molecular oscillator. The beauty of Landau and Teller's approach to an intrinsically complex kinetic phenomenon still impresses today's scientists and well deserves appreciation after 70 years.

\section{The Landau-Teller model: common trajectory approximation}

\subsection{Transition probabilities and encounter time}

Landau and Teller considered collinear collisions of a structureless particle A ("an atom" A) with a harmonic oscillator ("a diatomic molecule" BC) under the assumption that the vibrational amplitude is small compared to the range of the intermolecular forces and that the appropriately defined time $\tau$ (which we call the encounter time) is large compared to the inverse frequency of molecular oscillations. The potential energy surface (PES) used includes two coordinates, the distance $R$ between $\mathrm{A}$ and the centre of mass of $\mathrm{BC}$, and the interatomic distance $r$ in BC. Analytically, $U(R, r)$ for collinear $\mathrm{A}-\mathrm{BC}$ alignment, was taken as an exponential function of the A-B distance, $U(R, r) \propto \exp \left(-R_{\mathrm{AB}} / a\right)$, where $R_{\mathrm{AB}}=R-$ $\left(m_{\mathrm{B}} /\left(m_{\mathrm{A}}+m_{\mathrm{B}}\right)\right) r$ and $a$ is the length parameter that determines the steepness of the repulsion. For a non-vibrating diatom, with $r=r_{e}$, the interaction potential $U(R) \equiv U(R, r)_{r=r e}$ assumes a simple form $U(R)=C \exp (-R / a)$.

The following properties of this model were used to derive the dependence of the state-to-state $\left(n^{\prime} \rightarrow n^{\prime \prime}\right)$ rate coefficients $k_{n^{\prime} n^{\prime \prime}}(T)$ on the vibrational quantum numbers $n^{\prime}, n^{\prime \prime}$ of the oscillator and on the temperature $T$ of the heat bath:

(i) equidistant energy levels of the oscillator;

(ii) weak coupling of the oscillator (linear in $r-r_{e}$ ), to the translational mode, with a coupling interaction being proportional to the interaction potential between the atom $\mathrm{A}$ and the non-vibrating diatom $\mathrm{BC}$;

(iii) near-adiabatic conditions of the collision with respect to vibrational transitions.

The properties (i) and (ii) permit one, by the first-order timedependent perturbation approach, to derive the explicit features of the state-to-state probabilities $P_{n^{\prime}, n^{\prime \prime}}$ : transitions occur only between neighboring vibrational states, $n^{\prime \prime}=n^{\prime} \pm 1$, and $P_{n^{\prime}, n^{\prime \prime}}$ is proportional to $\max \left(n^{\prime}, n^{\prime \prime}\right)$. For instance, for the activating collision $n^{\prime} \rightarrow n^{\prime}+1$, the transition probability reads:

$$
P_{n^{\prime}, n^{\prime}+1}=\left(n^{\prime}+1\right) P_{0,1}\left(\omega, E_{\mathrm{t}}\right)
$$

where $E_{\mathrm{t}}$ is the relative translational energy of A and $\mathrm{BC}$ that specifies the time-dependent perturbation.

Property (iii) allows one to derive the dependence of the probability $P_{0,1}$ on the frequency. Landau and Teller outlined general arguments why the main dependence of the transition probability on $\omega$ for nearly adiabatic conditions will be exponential, i.e. is of the form

$$
P_{0,1}\left(\omega, E_{\mathrm{t}}\right) \propto \exp \left(-2 \omega \tau\left(E_{\mathrm{t}}\right)\right)
$$


The quantity $\tau\left(E_{\mathrm{t}}\right)$ measured with time units plays a key role in the theory of vibrational energy transfer. Landau and Teller also indicated how to calculate the time $\tau\left(E_{\mathrm{t}}\right)$ from the properties of a trajectory of relative motion in the field of the potential between the atom and non-vibrating diatom.

The rate coefficients $k_{n^{\prime}, n^{\prime}+1}(T)$ are expressed through the thermally averaged probabilities $\bar{P}_{n^{\prime}, n^{\prime}+1}(T)$ as

$$
k_{n^{\prime}, n^{\prime}+1}(T) \propto\left(n^{\prime}+1\right) \bar{P}_{0,1}(T)
$$

As shown by Landau and Teller, thermal averaging of $P_{1,0}\left(\omega, E_{\mathrm{t}}\right)$ for $\omega \tau \gg 1$ can be carried out in the steepest descent (SD) approximation which uses the interplay between a steep increase of $P_{1,0}\left(\omega, E_{\mathrm{t}}\right)$ with $E_{\mathrm{t}}$ and an exponential decrease of the Boltzmann factor with $E_{\mathrm{t}}$. In this way, the expression (3.2) for $P_{0,1}\left(\omega, E_{\mathrm{t}}\right)$ leads to

$$
\bar{P}_{0,1}(T) \propto \exp \left(-2 \omega \tau\left(E_{\mathrm{t}}^{*}\right)-E_{\mathrm{t}}^{*} / k_{\mathrm{B}} T\right)
$$

where the SD energy $E_{t}^{*}=E_{t}^{*}(\omega, T)$ is found from the equation

$$
\left.2 \omega \frac{\mathrm{d} \tau}{\mathrm{d} E_{\mathrm{t}}}\right|_{E_{\mathrm{t}}=E_{\mathrm{t}}^{*}}+\frac{1}{k_{\mathrm{B}} T}=0
$$

The actual calculation of $\tau$ for the exponential interaction yields: ${ }^{1}$

$$
\tau\left(E_{\mathrm{t}}\right)=\pi a \sqrt{\mu} / \sqrt{2 E_{\mathrm{t}}}
$$

where $\mu$ is the reduced mass of $\mathrm{A}$ and $\mathrm{BC}$.

The final result of eqns (3.1)-(3.6) can be summarized as

$$
\begin{aligned}
& k_{n^{\prime}, n^{\prime}+1}(T)=\left(n^{\prime}+1\right) k_{0,1}(T) \\
& k_{0,1}(T)=A_{0,1}(T) \exp \left(-3\left(T_{\mathrm{LT}} / T\right)^{1 / 3}\right)
\end{aligned}
$$

Here $T_{\mathrm{LT}}$ is the characteristic LT temperature

$$
T_{\mathrm{LT}}=\pi^{2} \omega^{2} \mu a^{2} / 2 k_{\mathrm{B}}
$$

and the pre-exponential factor $A_{0,1}(T)$ is supposed to depend on the temperature in a much weaker way than the exponential.

If one used the same arguments to derive expressions for rate coefficients for deactivating collisions $n^{\prime}+1 \rightarrow n^{\prime}$, one would find $k_{n^{\prime}+1, n^{\prime}}(T)=k_{n^{\prime}, n^{\prime}+1}(T)$. This result contradicts the detailed balance relation

$$
k_{n^{\prime}, n^{\prime}+1} / k_{n^{\prime}+1, n^{\prime}}=\exp \left(-\hbar \omega / k_{\mathrm{B}} T\right)
$$

and is caused by the basic shortcoming of the common trajectory (CT) approach. Landau and Teller realized the importance of detailed balance in formulating the relaxation equation, and they corrected this shortcoming of the CT approximation putting

$$
\begin{aligned}
& k_{n^{\prime}+1, n^{\prime}}(T)=\left(n^{\prime}+1\right) k_{1,0}(T) \\
& k_{1,0}(T)=A_{1,0}(T) \exp \left(-3\left(T_{\mathrm{LT}} / T\right)^{1 / 3}\right)
\end{aligned}
$$

where the pre-exponential factor $A_{1,0}(T)$ is related to that in eqn (3.7) in such a way that the relation (3.9) holds true: $A_{0,1}(T) / A_{1,0}(T)=\exp \left(-\hbar \omega / k_{\mathrm{B}} T\right)$. It is thus clear that the assumptions of weak temperature dependences of both $A_{0,1}(T)$ and $A_{1,0}(T)$ can be valid only under the condition $\hbar \omega / k_{\mathrm{B}} T \ll 1$ which was explicitly stated in the LT article. ${ }^{1}$

\subsection{Relaxation equations and Landau-Teller plot}

Landau and Teller constructed rate equations for the populations $N_{n}(t)$ of individual vibrational levels of BC molecules employing detailed balance arguments:

$$
\frac{\mathrm{d} N_{n}(t)}{\mathrm{d} t}=\sum_{n^{\prime} \neq n}[A] k_{n^{\prime}, n} N_{n^{\prime}}(t)-\left(\sum_{n^{\prime} \neq n}[A] k_{n, n^{\prime}}\right) N_{n}(t)
$$

The kinetic eqn (3.11) describes the relaxation of a system of oscillators to their equilibrium distribution

$$
N_{n}^{\mathrm{eq}}=\exp \left(-n \hbar \omega / k_{\mathrm{B}} T\right)\left(1-\exp \left(-\hbar \omega / k_{\mathrm{B}} T\right)\right)
$$

provided that the rate coefficients $k_{n, n^{\prime}}$ and $k_{n^{\prime}, n}$ satisfy the detailed balance relation.

An important feature of the LT rate equation for harmonic oscillators, eqn (3.11), using the rate coefficients from eqns (3.7) and (3.10), is that both the $k_{n, n+1}$ and the energy $E_{n}$ of the vibrational levels are linear in terms of the quantum number $n$. This made it possible to derive a closed equation for the mean vibrational energy $\bar{E}_{\mathrm{v}}(t)=\sum_{n} E_{n} N_{n}(t)$ of the molecules in the form:

$$
\frac{\mathrm{d} \bar{E}_{\mathrm{v}}(t)}{\mathrm{d} t}=-\frac{1}{\tau_{\mathrm{vib}}}\left(\bar{E}_{\mathrm{v}}(t)-\bar{E}_{\mathrm{v}}^{\mathrm{eq}}\right)
$$

where $\bar{E}_{\mathrm{v}}^{\mathrm{eq}}$ is the equilibrium thermal energy of the oscillator in the bath, and $\tau_{\text {vib }}$ is the vibrational relaxation time, such as given by

$$
\begin{aligned}
& \bar{E}_{\mathrm{v}}^{\mathrm{eq}}=\hbar \omega \exp \left(-\hbar \omega / k_{\mathrm{B}} T\right)\left(1-\exp \left(-\hbar \omega / k_{\mathrm{B}} T\right)\right)^{-1} \\
& 1 / \tau_{\mathrm{vib}}=[A] k_{1,0}\left(1-\exp \left(-\hbar \omega / k_{\mathrm{B}} T\right)\right)
\end{aligned}
$$

Eqn (3.13) represents an equation for the relaxation of a macroscopic quantity (the average energy of a molecular ensemble) derived from the master equation for the populations of individual levels. ${ }^{29-32}$ In many important applications, eqn (3.13) provided the missing link between gas-dynamics and kinetics and it was extensively used for the description of relaxation processes behind the front of shock waves.

Eqn (3.14) together with eqn (3.10) roughly predicts the temperature dependence of the vibrational relaxation time and of the $1 \rightarrow 0$ deactivation rate coefficient. If the temperature dependence arising from the last factor of the r.h.s. of eqn (3.14) as well as that arising from $A_{1,0}$ in eqn (3.10), are ignored, then the following relation holds

$$
-\ln \tau_{\mathrm{vib}} \propto \ln k_{10}=\mathrm{const}-3\left(T_{\mathrm{LT}} / T\right)^{1 / 3}
$$

where the LT temperature is defined by eqn (3.8). Eqn (3.15) forms the basis of the frequently used Landau-Teller plot.

The following comments on some issues that are directly related to the LT model and which are of interest for the present article are in order:

(i) The exponential dependence of the transition probability on the frequency is the a consequence of the Ehrenfest adiabaticity principle and it is explained in detail within the context of classical mechanics (see, e.g., ref. 22). It is analogous to a similar dependence of the change in the vibrational energy of classical oscillators. In the latter case, the near adiabatic condition $\omega \tau \gg 1$ ensures that the change in the vibrational energy is small and, therefore, a feedback of the 
oscillatory mode on the relative motion can be neglected. This allows one to identify the collision energy $E_{\mathrm{t}}$ with the initial or final translational energy, $E_{t}^{\prime}$ or $E_{t}^{\prime \prime}$. For a quantum oscillator, the adiabatic condition $\omega \tau \gg 1$ guarantees a small change in the mean vibrational energy, though the individual energy transfer $\Delta E=\hbar \omega$ may not be small. Then the collision energy $E_{\mathrm{t}}$ can be affected by $\Delta E$, though in a way yet unknown.

(ii) Vibrational transitions with exponentially low transition probabilities are classically forbidden, implying that a purely classical solution of the collision problem will not bring the oscillator from an energy level $E_{n}$ to a neighboring level $E_{n \pm 1}$. Nonetheless, transition probabilities are calculated with a classical description of the relative motion. This is possible due to the correspondence principle, asserting that the matrix element of a physical quantity $V(R), V_{E_{\mathrm{t}}{ }^{\prime} E_{\mathrm{t}}{ }^{\prime \prime}}$, between closelylying semiclassical states (in the WKB sense) with energies $E_{\mathrm{t}}{ }^{\prime}$ and $E_{t}^{\prime \prime}$ is equal to the Fourier component of the time dependent quantity, $V\left(R_{E_{\mathrm{t}}}(t)\right)$, calculated for the transition frequency $\omega=\left(E_{\mathrm{t}}{ }^{\prime}-E_{t}^{\prime \prime}\right) / \hbar$ along a trajectory $R_{E_{\mathrm{t}}}(t)$ that corresponds to a certain mean (but not well defined) energy $E_{\mathrm{t}}$ (the essence of the CT approximation), see ref. 23.

(iii) Transition probabilities are calculated in the first order in the diabatic basis, i.e. in the basis of unperturbed vibrational states of an oscillator. The LT problem contains two parameters for estimating the higher-order corrections, a small coupling parameter $\eta$ that enters the factor $A$ in eqn (3.7) and the large Massey parameter $\Xi \propto \omega \tau$ that enters the exponential in eqn (3.2). The first order diabatic (FOD) term of the perturbation series in powers of $\eta$ roughly reads $P^{\mathrm{FOD}} \propto \eta$ $\exp (-\Xi)$. The most important second-order diabatic (SOD) correction will come as a modification not of the pre-exponential factor, but of the exponent, $P^{\mathrm{SOD}} \propto \eta \exp (-\Xi(1+\eta))$. Since $\Xi \gg 1$, the product $\Xi \eta$ may not be small, and can, therefore, noticeably affect the first-order result. This effect, the early breakdown of FOD in vibrational energy transfer, was studied in ref. 24-28. It was shown that the first-order treatment in the adiabatic basis (FOA) to a large extent incorporates higher order corrections in the diabatic basis.

(iv) The classical limit $\left(\hbar \omega / k_{\mathrm{B}} T \ll 1\right)$ of eqn (3.14) sheds some light on the features of the CT approximation. The mean energy transferred to the oscillator initially in its ground state under the action of an external force is $\left.\Delta \bar{E}_{\mathrm{v}}^{\mathrm{LT}}\right|_{n=0}=\hbar \omega \bar{P}_{01}^{\mathrm{LT}}$. It turns out that $\left.\Delta \bar{E}_{\mathrm{V}}^{\mathrm{LT}}\right|_{n=0}$ equals the mean classical energy transferred to the oscillator initially at rest, $\left.\Delta \bar{E}_{\mathrm{v}}^{\mathrm{Cl}}\right|_{E_{\mathrm{v}}=0}=$ $\Delta \bar{E}_{0}^{\mathrm{Cl}}$. This permits one to recover the LT transition probability from the classical energy transfer, $\bar{P}_{01}^{\mathrm{LT}}=\Delta \bar{E}_{0}^{\mathrm{Cl}} / \hbar \omega$, and to rewrite the single-collision classical counterpart of the relaxation eqn (3.11) as an increment $\Delta \bar{E}_{\mathrm{v}}^{\mathrm{Cl}}\left(E_{\mathrm{v}}\right)$ in the initial vibrational energy $E_{\mathrm{v}}$ after a collision, $\Delta \bar{E}_{\mathrm{v}}^{\mathrm{Cl}}\left(E_{\mathrm{v}}\right)=\Delta \bar{E}_{0}^{\mathrm{Cl}}[1-$ $\left.E_{\mathrm{v}} / k_{\mathrm{B}} T\right]$. This equation predicts a decrease or an increase in the vibrational energy of the oscillator after a collision, depending on whether its initial vibrational energy is above or below the equilibrium energy. The classical dynamical counterpart of the above expression can be obtained as the phase-averaged classical energy transfer to the vibrating oscillator under the action of an external force. In the standard approach within the CT approximation, one assumes a uniform distribution of the phase $\varphi$ at the maximum of the perturbing force, which yields $\left\langle\Delta E_{\mathrm{v}}^{\mathrm{Cl}}\left(E_{\mathrm{v}}\right)\right\rangle_{\varphi}=\Delta \bar{E}_{0}^{\mathrm{Cl}}$. This result predicts an increase of the vibrational energy after each collision that is wrong. Attempts to derive the correct expression for the mean energy transfer from the dynamical equations require getting beyond the CT approximation, which amounts to more accurate considerations of the phase averaging. ${ }^{33,34}$ On the other hand, the purely dynamical calculation of the mean-squared energy transfer in the CT approximation with a uniform distribution of $\varphi$ and under the condition $E_{\mathrm{v}} \gg \Delta \bar{E}_{0}^{\mathrm{Cl}}$ yields $\left\langle\left(\Delta E_{\mathrm{v}}^{\mathrm{Cl}}\left(E_{\mathrm{v}}\right)\right)^{2}\right\rangle_{\varphi}$ $=2 E_{\mathrm{v}} \Delta \bar{E}_{0}^{\mathrm{Cl}}$, which is in agreement with the result that can be obtained from the kinetic eqn (3.11). One can, therefore, use the dynamical classical CT mean-squared energy transfer as the diffusion coefficient in the Fokker-Planck-Kramers ${ }^{35}$ relaxation equation, which incorporates the detailed balancing by introducing the Einstein relation between diffusion and mobility coefficients. The single collision version of the Kramers equation yields, of course, correct expression for the mean energy transfer.

(v) Finally, we comment on the correction of the CT transition probabilities to comply with the detailed balance relation. This question attracted attention mainly in connection with the recovery of the quantum correlation function of the bath from its classical counterpart (see the review of ref. 36 and the recent articles ref. 37-44; in particular, ref. 44 provides a list of the different factors which have been recommended to correct the classical correlation function). We will address this issue in section 4 .

\section{Semiclassical generalization of the common trajectory model}

\subsection{The Landau exponent and the Landau-Teller time}

The semiclassical generalization of the CT LT collinear model in this section is discussed on the basis of the recovery of the Landau semiclassical (SC) exponent from the LT classical encounter time. (In what follows we will use the term "semiclassical" as "satisfying the WKB conditions". In the textbook by Landau and Lifshitz, ${ }^{23}$ as well as in the Russian literature on quantum mechanics, states that satisfy the WKB conditions are called "quasiclassical". However, since in the context of molecular collision theory the notion "quasiclassical" is widely used in connection with purely classical trajectory calculations run with quantum initial conditions, we adhere in this paper to "semiclassical".) In 1932, Landau ${ }^{14}$ formulated a general SC method for the calculation of matrix elements and probabilities under the condition that these quantities are exponentially small. This method, as associated with the WKB approximation, is outlined in the textbook by Landau and Lifshitz. ${ }^{23}$

According to the SC Landau method, ${ }^{14}$ the exponential part of the probability for transition between states with translational energies $E_{\mathrm{t}}^{\prime}$ and $E_{\mathrm{t}}^{\prime \prime}=E_{\mathrm{t}}^{\prime}+\Delta E$ reads

$$
P^{\mathrm{SC}}\left(E_{\mathrm{t}}^{\prime}, \Delta E\right) \propto \exp \left(-(2 / \hbar)\left|\operatorname{Re}\left(\tilde{S}_{E_{\mathrm{t}}^{\prime}}-\tilde{S}_{E_{\mathrm{t}}^{\prime}+\Delta E}\right)\right|\right)
$$

In eqn (4.1), $\tilde{S}_{E_{\mathrm{t}}^{\prime}}$ (or $\tilde{S}_{E_{\mathrm{t}}^{\prime}}+\Delta E$ ) is the classical action integral taken from the turning point for the motion across the unperturbed potential at the energy $E_{\mathrm{t}}^{\prime}\left(\right.$ or $E_{\mathrm{t}}^{\prime \prime}=E_{\mathrm{t}}^{\prime}+\Delta E$ ) into the classically forbidden region of this potential to a transition distance determined by the stationary phase 
condition for the action difference. (In a more sophisticated version of the SC approach, the exponent in the expression for the transition probability, besides the difference in the classical actions integrals, also depends on certain properties of the perturbation. ${ }^{23}$ This approach in its application to vibrational transitions is discussed in ref. 45, but will not be touched here.) Since the SC transition probability is roughly proportional to the square of the overlap of the initial and final WKB wave functions, it is tempting to interpret the exponential in eqn (4.1) as the result of an overlap of WKB functions in the classically forbidden region of motion. However, this is not correct, since the overlap would contain the sum and not the difference of the classical actions. Though an excursion into the classically forbidden region of the potential looks like a completely nonclassical event, this is not quite so for potentials that are represented by analytical functions, since the full potential can be recovered from a small portion of it and, therefore, the potential in the classically forbidden region is completely defined by the potential in a classically allowed region. One, therefore, would hope to find a relation between the classical exponent in eqn (3.2) and the semiclassical exponent in eqn (4.1). Indeed, expression (4.1) can be recast in the form: ${ }^{15,16}$

$$
P_{10}^{\mathrm{SC}}\left(E_{\mathrm{t}}^{\prime}, \Delta E\right) \propto \exp \left(-(2 / \hbar)\left|\int_{E_{\mathrm{t}}^{\prime}}^{E_{\mathrm{t}}^{\prime}+\Delta E} \tau(E) \mathrm{d} E\right|\right)
$$

which results in the following expression for the thermallyaveraged probability

$$
\bar{P}_{10}^{\mathrm{SC}}(T, \Delta E) \propto \int \exp \left(-(2 / \hbar)\left|\int_{E_{\mathrm{t}}^{\prime}}^{E_{\mathrm{t}}^{\prime}+\Delta E} \tau(E) \mathrm{d} E\right|-\frac{E_{\mathrm{t}}^{\prime}}{k_{\mathrm{B}} T}\right) \frac{\mathrm{d} E_{\mathrm{t}}^{\prime}}{k_{\mathrm{B}} T}
$$

that clearly complies with the detailed balance relation, $\bar{P}_{10}^{\mathrm{SC}}(T, \Delta E) / \bar{P}_{10}^{\mathrm{SC}}(T, \Delta E)=\exp \left(\hbar \omega / k_{\mathrm{B}} T\right)$

Since the exponent in eqn (4.2) is completely determined by the classical time $\tau(E)$ we dwell on the question how this quantity can be calculated. According to Landau and Teller, the time $\tau(E)$ is defined through the integral:

$$
\tau\left(E_{\mathrm{t}}\right)=\operatorname{Im}\left\{\int_{R_{\mathrm{s}}}^{R_{\mathrm{t}}} \frac{\mathrm{d} R}{\sqrt{(2 / \mu)\left(E_{t}-U(R)\right)}}\right\}
$$

Here $U(R)$ is the unperturbed interaction potential between $\mathrm{A}$ and the centre of mass of $\mathrm{BC}, R_{\mathrm{t}}$ is the turning point for the motion in the field of the potential $U(R)$, and $R_{\mathrm{s}}$ is a singular point of the coupling interaction. The detailed discussion of eqn (4.6) can be found in ref. 50. Here we only mention that though originally the time $\tau(E)$ was defined for asymptotically free relative motion of $\mathrm{A}$ and $\mathrm{BC}$, for $E=E_{\mathrm{t}}>0$, eqn (4.4) permits a generalisation for the bound motion in the potential well of the complex $\mathrm{A}-\mathrm{BC}$, when the energy $E$ acquires the meaning of the vibrational energy of the $\mathrm{A}-\mathrm{BC}$ bond. This implies that eqn (4.2) can be applied not only to free-free transition (vibrational-translational energy transfer, both $E^{\prime}$ and $E^{\prime \prime}$ are positive), but also to bound-free transitions (vibrational predissociation, negative $E^{\prime}$ and positive $E^{\prime \prime}$ ) and bound-bound transitions (intramolecular energy redistribution, both $E^{\prime}$ and $E^{\prime \prime}$ are negative). In the whole range of energies, starting from the bottom of the well, the function $\tau(E)$ decreases monotonically. An analytical example is given by the encounter time for a Morse potential. ${ }^{45,46}$

\subsection{Restoring detailed balance in the CT approximation}

A consistent correction of the CT approximation complying with the detailed balance relation can be derived from the SC approximation. To this end we consider small energy transfer such that $\tau(E)$ can be approximated by its linear expansion

$$
\tau(E)=\tau\left(E_{\mathrm{t}}^{\prime}\right)+\frac{\mathrm{d} \tau\left(E_{\mathrm{t}}^{\prime}\right)}{\mathrm{d} E_{\mathrm{t}}^{\prime}}\left(E-E_{\mathrm{t}}^{\prime}\right)
$$

With this approximation we get

$$
\begin{aligned}
& \left.\bar{P}^{\mathrm{SC}}(T, \Delta E)\right|_{|\Delta E| \text { small }} \propto \\
& \int \exp \left(-2 \omega \tau\left(E_{t}^{\prime}\right)-\frac{E_{\mathrm{t}}^{\prime}}{k_{\mathrm{B}} T}-\omega \Delta E \frac{\mathrm{d} \tau\left(E_{\mathrm{t}}^{\prime}\right)}{\mathrm{d} E_{\mathrm{t}}^{\prime}}\right) \frac{\mathrm{d} E_{\mathrm{t}}^{\prime}}{k_{\mathrm{B}} T}
\end{aligned}
$$

where the last term in the exponent is assumed to be small compared to the other two terms. Then the SD energy is determined by the first two terms such as given by eqn (3.5). In this way, we finally obtain the relation between the CT and corrected CT (CCT) transition probabilities, with the latter defined as SC transition probabilities for small $|\Delta E|$ :

$$
\begin{aligned}
\left.\bar{P}^{\mathrm{SC}}(T, \Delta E)\right|_{\text {small }|\Delta E|} & \equiv \bar{P}^{\mathrm{CCT}}(T, \Delta E) \\
& =\exp \left(-\Delta E / 2 k_{\mathrm{B}} T\right) \bar{P}^{\mathrm{CT}}(T, \Delta E)
\end{aligned}
$$

where $\Delta E>0$ for up-transitions and $\Delta E<0$ for downtransitions, and $\bar{P}^{\mathrm{CT}}(\Delta E)$ does not depend on the sign of $\Delta E$. The relation between the CCT and CT expressions in the form of eqn (4.7) was first established by Schwartz, Slawsky and Herzfeld in $1952,{ }^{4}$ for the special case of an exponentially repulsive interaction, by analyzing the near-adiabatic SC limit of the quantum expression derived by Jackson and Mott. ${ }^{5}$ However, Schwartz, Slawsky and Herzfeld did not identify the leading term in the exponent of the expression for $\left.\bar{P}^{\mathrm{SC}}(T, \Delta E)\right|_{\text {small } \mid \Delta E}$ with the LT exponent, such that their approach did not, strictly speaking, provide a correction to the LT probability. This unfortunate initial misunderstanding, however, was corrected later in ref. 7. In 1983, Miklavc ${ }^{47}$ repeated the use of the Jackson-Mott solution for establishing the corrected form of the average transition probability. Most generally and valid for arbitrary interactions, the correction factor appearing in eqn (4.7) was suggested by Nikitin in $1959^{48}$ from symmetry considerations and by Schofield in $1960^{49}$ on the basis of an analytical continuation of the classical correlation function. It should be noted, however, that the correction term in eqn (4.7) differs from respective quantum correction factors derived by other authors, such as those listed in Table 1 of ref. 44. The differences are due to different conditions under which the various corrections are valid, and to different high-frequency limits of the Fourier transform of the force correlation function of different heat baths. 
The correction factor in eqn (4.7) does not depend on the property of the heat bath except its temperature. At the same time, this factor represents the first quantum correction to the classical correlation function of the external force acting on the oscillator. The influence of higher-order corrections on the transition probabilities can be estimated by identifying the expansion parameter in the truncated series in eqn (4.9). ${ }^{50}$ With this correction, the temperature dependence of the CCT deactivation probability reads

$$
\begin{aligned}
\ln \bar{P}_{10}^{\mathrm{CCT}}= & \text { const }-3 \Xi(\omega, T)+\frac{\hbar \omega}{2 k_{\mathrm{B}} T} \\
& +O\left(\frac{\left(\hbar \omega / k_{\mathrm{B}} T\right)^{2}}{\Xi(\omega, T)}\right)
\end{aligned}
$$

where $3 \Xi(\omega, T)=2 \omega \tau\left(E_{t}^{*}\right)+E_{t}^{*} / k_{\mathrm{B}} T, E_{t}^{*}$ is found as the solution of the SD condition (3.5), and where $O(x)$ means "of the order of $x$ ". It follows from eqn (4.8) that the condition of small energy transfer $\Delta E=\hbar \omega$, as appropriate for the CCT approximation, is formulated as $\left(\Delta E / k_{\mathrm{B}} T\right)^{2} \ll \Xi(\omega, T)$. Since in the near-adiabatic regime $\Xi(\omega, T) \gg 1$, the latter inequality permits the ratio $|\Delta E| / k_{\mathrm{B}} T$ to fall into the quantum range when $\hbar \omega / k_{\mathrm{B}} T \geq 1$.

Since the LT plot is based on a repulsive potential, it is appropriate to explicitly identify also the correction that comes from an attractive part of the interaction. For a Morse or Lennard-Jones potential, for which the attractive part is proportional to the square root of the repulsive part, the logarithmic representation of $k_{10}(T)$ in the CCT approximation reads

$$
\begin{aligned}
\ln \bar{P}_{10}^{\mathrm{CCT}} & =\text { const }-3 \Xi_{\mathrm{rep}}(\omega, T)+\frac{\hbar \omega}{2 k_{\mathrm{B}} T} \\
& +c \sqrt{\frac{D}{k_{\mathrm{B}} T} \Xi_{\mathrm{rep}}(\omega, T)}+O\left(\frac{\left(\hbar \omega / k_{\mathrm{B}} T\right)^{2}}{\Xi_{\mathrm{rep}}(\omega, T)}\right) \\
& +O\left(\frac{D}{k_{\mathrm{B}} T}\right)
\end{aligned}
$$

where $c$ is a numerical coefficient. Here $3 \Xi_{\text {rep }}(\omega, \tau)=2 \omega \tau_{\text {rep }^{-}}$ $\left(E^{*}\right)+E^{*} / k_{\mathrm{B}} T$ and $E^{*}$ is found from the SD condition $2 \omega-$ $\left.\left(\mathrm{d} \tau_{\mathrm{rep}} / \mathrm{d} E\right)\right|_{E=E^{*}}+1 / k_{\mathrm{B}} T=0$ with the encounter time $\tau_{\text {rep }}=$ $\tau_{\text {rep }}(E)$ calculated for the repulsive, high energy, part of the interaction. This expression provides several reasons for curvatures of Landau-Teller plots: (i) deviations of the repulsive part from purely exponential form; (ii) attraction at larger distances; and (iii) quantum effects of the energy release. For a purely repulsive potential of the form $U(R) \propto R^{-n}$, one has $\Xi_{\text {rep }}(T) \propto T^{-(n+2) /(3 n+2)}$ (for reasons (i), for a Morse potential $\Xi_{\text {rep }}(T, \omega) \propto T^{-1 / 3}$ and $c=4 / \pi$, (for reasons (ii) and (iii), while for a Lennard-Jones potential $\Xi_{\text {rep }}(T) \propto T^{-7 / 19}$ and $c=4.44 / \pi$ (for all three reasons). ${ }^{51,52}$ We note that the correction for the attraction (the third term in eqn (4.9)) is larger than that suggested in ref. 4 , namely $D / k_{\mathrm{B}} T$. The reason for this is that the main manifestation of the attraction is the increase in the steepness of the interaction at lower energies rather than an additional acceleration of the partners when they pass the potential well. ${ }^{48}$

\subsection{Dependence of the SC transition probabilities on the energy release in special cases}

The dependence of the SC transition probability on the energy release $\Delta E$ is determined by eqn (4.2). In the following, we discuss approximations to the Landau exponent which are currently used in some applications.

4.3.1 Symmetrization of common trajectories. In an attempt to extend the multidimensional CT method beyond its CCT version, a symmetrization of the initial parameters of the trajectory with respect to the initial and final quantum states of the oscillator was suggested. We exemplify this approach for a single bath variable. In general, one can write

$$
(2 / \hbar)\left|\int_{E_{\mathrm{t}}^{\prime}}^{E_{\mathrm{t}}^{\prime}+\Delta E} \tau(E) \mathrm{d} E\right|=2 \omega \tau(\bar{E})
$$

where $\bar{E}$ is a certain energy bracketed between $E_{\mathrm{t}}^{\prime}$ and $E_{\mathrm{t}}^{\prime \prime}$ with the function $\bar{E}=\bar{E}\left(E_{\mathrm{t}}^{\prime}, E_{\mathrm{t}}^{\prime \prime}\right)$ being symmetric with respect to the exchange of $E_{\mathrm{t}}^{\prime}$ and $E_{\mathrm{t}}^{\prime \prime}$. It is seen that this representation of the integral introduces the quantum quantity $\hbar \omega=\left|E_{\mathrm{t}}^{\prime}-E_{\mathrm{t}}^{\prime \prime}\right|$ into the classical collision time, and makes the semiclassical exponent look like an "effective" classical exponent calculated for a common trajectory with the initial energy $\bar{E}$. This approach is the basis for the so-called quantum-classical method by Billing. ${ }^{53}$ Since the function $\bar{E}=\bar{E}\left(E_{\mathrm{t}}^{\prime}, E_{\mathrm{t}}^{\prime \prime}\right)$ is not known a priori, one can try different choices to reduce the errors introduced by the standard CT approximation. The two simplest ways would be to symmetrize the initial energy or the initial velocity by putting

$$
\begin{gathered}
\bar{E}=(1 / 2)\left(E_{\mathrm{t}}^{\prime}+E_{\mathrm{t}}^{\prime \prime}\right) \\
\bar{E}=\left(\sqrt{E_{\mathrm{t}}^{\prime}}+\sqrt{E_{\mathrm{t}}^{\prime \prime}}\right)^{2} / 4
\end{gathered}
$$

An interesting feature of the symmetrization in accord with eqn (4.12) is that, if applied to the case of an exponential repulsive interaction, it makes the "classical" quantity $2 \omega \tau(\bar{E})$ exactly equal to its semiclassical counterpart $(2 / \hbar)\left|\int_{E_{\mathrm{t}}^{\prime}}^{E_{\mathrm{t}}^{\prime}+\Delta E} \tau(E) \mathrm{d} E\right|$. This might be the reason why the classical-quantum approach proved to be so successful in applications to vibrational relaxation events even when the interaction potential differed to some extent from an exponential form.

4.3.2 Energy gap regime for the collisional relaxation of an anharmonic oscillator. The collision-induced relaxation of anharmonic oscillators in a heat bath provides an example for the manifestation of the so-called energy gap law.

According to eqn (4.9), the Landau exponent in the CCT approximation reads

$$
(2 / \hbar)\left|\int_{E_{\mathrm{t}}^{\prime}}^{E_{\mathrm{t}}^{\prime}+\Delta E} \tau(E) \mathrm{d} E\right|=\frac{|\Delta E|}{\hbar}\left(2 \tau\left(E_{\mathrm{t}}^{\prime}\right)+\Delta E \frac{\mathrm{d} \tau\left(E_{\mathrm{t}}^{\prime}\right)}{\mathrm{d} E_{\mathrm{t}}^{\prime}}\right)
$$


In this equation, the second term at the r.h.s. ensures detailed balancing under the condition $|\Delta E| \ll E_{\mathrm{t}}^{\prime}$. Since the main dependence of the transition probability on the energy transfer $\Delta E$ comes from the first term, $P^{\mathrm{CCT}}\left(E_{\mathrm{t}}^{\prime}, \Delta E\right)$ will be nearly exponential in $|\Delta E|$ which corresponds to the so-called energy gap law. In the following we use eqn (4.13) for the calculation of thermally averaged transition probabilities for anharmonic oscillators.

The anharmonicity of the oscillator affects eqns (3.7) and (3.10) in two ways: the matrix elements for the oscillator coordinate allow for transitions with $\Delta n>1$, and the energy change for a transition $n \rightarrow n+\Delta n$ depends on $n$. In the first attempt to account for anharmonicity, it was assumed that, due to a small change in the frequency with vibrational energy, the transition probabilities $\bar{P}_{n, n}+\Delta n$ would be proportional to $\bar{P}_{01}^{\mathrm{LT}}$ with the prefactors proportional to $\langle n|x| n+\Delta n\rangle^{2} \cdot{ }^{54}$ This generalization turned out to be incorrect for two reasons: first, the transition frequency for $\Delta n>1$, being roughly a multiple of $\omega$, makes the exponential factor negligibly small compared to that with $\Delta n=1$, and second, a small variation of the transition frequency with $n$ affects the exponential factor in the expression for the transition probability by far more strongly than the pre-exponential factor. ${ }^{55}$ Thus, a generalization of the LT eqns (3.7) and (3.10) for anharmonic oscillators (AO) still conserves the single-quantum character of the energy transfer, but modifies the transition probabilities. For instance, for an anharmonic oscillator with a standard expression for the vibrational energy $E_{n}=\hbar \omega_{e}(n+1 / 2)-x_{e} \hbar \omega_{e}(n+1 / 2)^{2}$, the transition probabilities in the CCT approximation will read

$$
\begin{aligned}
& \bar{P}_{n+1, n}^{\mathrm{CCT}-\mathrm{AO}}=(n+1) b^{n+1} \bar{P}_{10}^{\mathrm{CCT}-\mathrm{HO}} \\
& \bar{P}_{n, n+1}^{\mathrm{CCT}-\mathrm{AO}}=\bar{P}_{n+1, n}^{\mathrm{CCT}-\mathrm{AO}} \exp \left(-\left(\hbar \omega_{e}-2 x_{e} \hbar \omega_{e} n\right) / k_{\mathrm{B}} T\right) \\
& \bar{P}_{n, n^{\prime}}^{\mathrm{CCT}-\mathrm{AO}}=0, \quad \text { if } n^{\prime} \neq n \pm 1
\end{aligned}
$$

where $b=\exp \left(\left(T_{\mathrm{LT}} / T\right)^{1 / 3} x_{e}\right)$ and $\bar{P}_{10}^{\mathrm{CCT}-\mathrm{HO}}$ is the CCT transition probability for a harmonic oscillator calculated with a small-amplitude frequency $\omega_{e}$. Thus the anharmonicity changes the linear dependence of the neighbouring transition probabilities on $n$ into a superlinear dependence.

4.3.3 The momentum gap regime for the relaxation of anharmonic oscillators in vibrational predissociation. Dissociation-induced relaxation (vibrational predissociation, VP) of an anharmonic oscillator $\mathrm{BC}$ in a van-der-Waals complex A $\cdots \mathrm{BC}$ provides an example for the manifestation of the so-called momentum gap law.

A detailed model of vibrational predissociation for a twomode model (high-frequency anharmonic oscillator coupled to a low-frequency dissociative Morse oscillator) was studied by Beswick and Jortner ${ }^{56-58}$. Analytical expressions that describe the dependence of the VP rate on the parameters of a $\mathrm{A}-\mathrm{BC}$ system were obtained.

The Landau method suggests an alternative approach which can be used for arbitrary potentials, though being restricted by the SC conditions of motion in the initial and final states. Usually, when the energy transferred to the dissociating oscillator, $\Delta E$, is large compared to the dissociation energy, the final state is semiclassical. The initial state may not be semiclassical in the global WKB sense, but it is then nearly harmonic. The latter property ensures that the tunneling asymptotics of the initial wave function is very close to its WKB counterpart ${ }^{59}$ and, therefore, the Landau formula eqn (4.2) for the SC transition probability provides a good approximation to accurate quantum results. The SC probability for the dissociation of a low-frequency oscillator per one vibration in its initial state with the vibrational energy $E^{\prime}$, induced by a vibrational transition $n \rightarrow n-1$ in the highfrequency oscillator, $P_{n, n-1}^{\mathrm{SC}-\mathrm{VP}}\left(E^{\prime}\right)$, can be written as

$$
P_{n, n-1}^{\mathrm{SC}-\mathrm{vP}}\left(E^{\prime}\right) \propto \exp \left(-\frac{2}{\hbar} \int_{E^{\prime}}^{E^{\prime}+\Delta E_{n, n-1}} \tau(E) \mathrm{d} E\right)
$$

If $\Delta E_{n, n-1}$ markedly exceeds the dissociation energy $D$ of the complex, the recoil energy of the fragments A and BC (relative translational energy $E_{t}^{\prime \prime}$ ) approximately equals the energy release $\Delta E_{n, n-1}$ and the integral in eqn (4.15) is dominated by the repulsive region of interaction. Taking the latter to be roughly exponential and putting $\tau(E) \approx \pi a / 2 E / \mu$ we get an estimate

$$
\left.\frac{2}{\hbar} \int_{E_{\mathrm{v}}^{\prime}}^{E_{\mathrm{v}}^{\prime}+\Delta E_{n, n-1}} \tau(E) \mathrm{d} E\right|_{D \ll \Delta E_{n, n-1}} \approx \frac{2 \pi a}{\hbar} \sqrt{2 \mu E_{\mathrm{t}}^{\prime \prime}}
$$

The expression in eqn (4.15) in conjunction with eqn (4.16)) is called the momentum gap law: ${ }^{60}$ the VP rate decreases exponentially with an increase in the relative momentum of receding fragments, $p^{\prime \prime} \approx \sqrt{2 \mu E_{\mathrm{t}}^{\prime \prime}}$. We note in passing that eqns (4.15) and (4.16) have only little relation to the Morse potential of the dissociative oscillator, though, of course, they can be derived from this model ${ }^{56-58}$ and put in such a way that the length parameter $a$ is expressed through the dissociation energy and the frequency of oscillation of the van der Waals bond. Theoretical predictions from eqns (4.15) and (4.16) can be tested against experiment in two ways: by considering the dependence of the predissociation rate either on the energy release for different systems at a fixed $n$, or by studying the dependence of the rate on $n$ for the same system. The former correlation was presented by Eving ${ }^{60}$ as a linear plot of the logarithm of predissociation lifetimes for different systems $v s$. $a \sqrt{ } 2 \mu \Delta E / \hbar$ with an empirical fitting of the value of $a$. The latter correlation can be deduced from $\Delta E_{n, n-1}$ for an anharmonic oscillator, $\Delta E_{n, n-1}=\hbar \omega_{e}\left(1-2 x_{e} n\right),{ }^{61}$ once it is realised that the unspecified pre-exponential factor in eqn (4.18) is proportional to $n$ (similar to that for the collisional relaxation of anharmonic oscillator (vide supra)). In this way one can write the following expression for the $n$-dependence of the VP rate:

$$
\begin{gathered}
k_{n, n-1}^{\mathrm{VP}} \propto P_{n, n-1}^{\mathrm{SC}-\mathrm{VP}} \propto n \exp (\lambda n) \\
\lambda=2 \pi x_{e} a \sqrt{2 \mu \omega_{e} / \hbar}
\end{gathered}
$$

Fig. 1, for a wide range of vibrational quantum numbers $n$, illustrates the super-linear dependence on $n$ of the vibrational deactivation rate in the VP event for the decay of $\mathrm{vdW}$ complexes $\mathrm{I}_{2}\left(\mathrm{~B}^{3} \Pi_{0}, n\right)-\mathrm{Ne} \rightarrow \mathrm{I}_{2}\left(\mathrm{~B}^{3} \Pi_{0}, n-1\right)+\mathrm{Ne}$. 


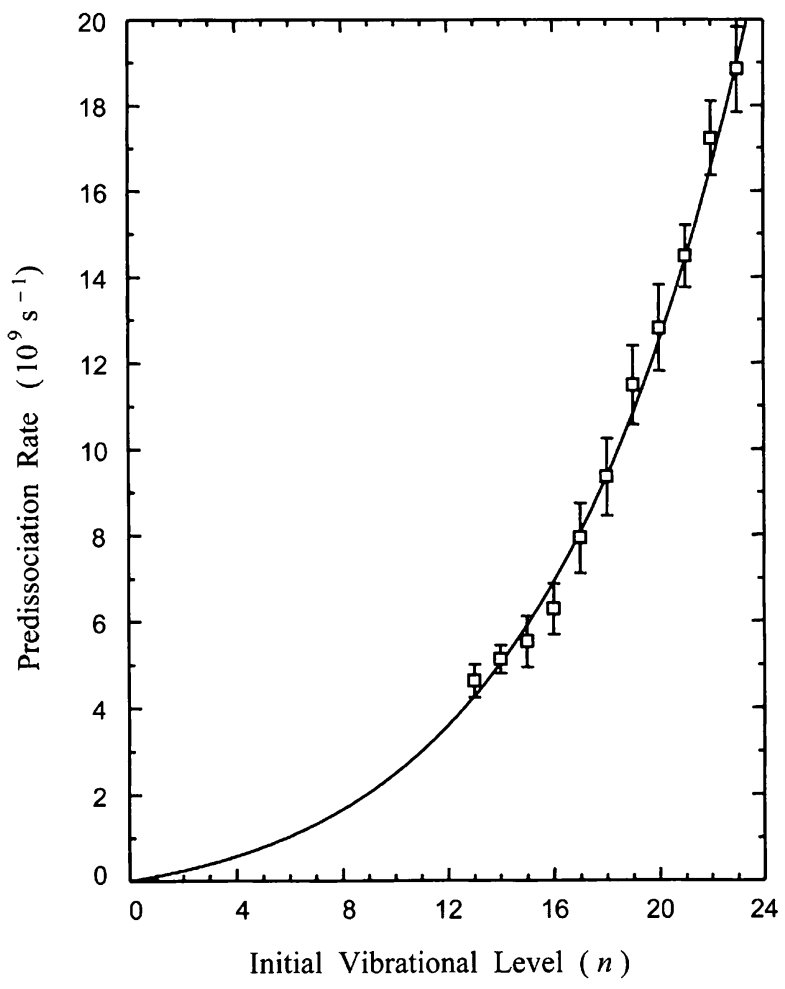

Fig. 1 Example of a super-linear dependence of the vibrational predissociation rate on the vibrational quantum number $n$. Squares are experimental data for $\mathrm{I}_{2}\left(\mathrm{~B}^{3} \Pi_{0}, n\right)-\mathrm{Ne} \rightarrow \mathrm{I}_{2}\left(\mathrm{~B}^{3} \Pi_{0}, n-1\right)+\mathrm{Ne}$ predissociation, full line represents theoretical fitting. The deviation from a straight line (Landau-Teller eqn (3.10)) is due to the dependence of the transition probability on the energy release in the limit of the momentum gap law, see eqn (4.15) (after ref. 61).

Different from vibrational relaxation which occurs in successive collisions of $\mathrm{BC}$ molecules with heat bath atoms $\mathrm{A}$, the kinetics of which is described by the LT eqn (3.11) or its diffusion Kramers counterpart, the vibrational predissociation occurs in a "half-collision". However, the SC picture shows that VP occurs, with low probability, each time when the partners visit the repulsive branch of the potential. Thus it became a challenge to also study the classical counterpart of VP, i.e. the dissociation of a classical low-frequency dissociative oscillator coupled to a high-frequency mode (another oscillator or a periodic time-dependent external field. ${ }^{62-74}$

\section{Three-dimensional generalizations of the semiclassical collinear model}

Three-dimensional collisions of an atom with a non-vibrating diatomic molecule in an adiabatically isolated electronic state occur on a potential energy surface $U(R, \gamma)$. The two coordinates, that are conventionally chosen, are the separation $R$ between $\mathrm{A}$ and the centre of mass of the rotor BC, and the polar angle $\gamma$ between the rotor axis $\boldsymbol{r}$ and the collision axis $\boldsymbol{r}$. Once the conservation of the total angular momentum $\boldsymbol{J}$ is taken into account, the Hamiltonian of the A + BC (rigid rotor) system in the body-fixed frame contains three coordinates and conjugate momenta. Besides $R$ and $\gamma$, the kinetic energy depends on the azimuthal angle $\varphi$ of $\boldsymbol{r}$ in the plane normal to $\boldsymbol{r}$. The part of the kinetic energy that depends on $\varphi$, describes the Coriolis interaction in the $\mathrm{BF}$ coordinate frame. Three-dimensional generalizations of the SC collinear model are based on the transformation of these variables in such a way that only one of these, to be called the driving mode, is essentially coupled to the vibration of BC. This transformation uses the hierarchy of parameters that exists in the problem of the vibrational relaxation under near-adiabatic conditions. Following Ovchinnikova, ${ }^{75}$ for diagnostic purposes, we introduce two encounter times, one for the radial motion, $\tau_{\mathrm{tr}}$, and the other for the angular motion, $\tau_{\text {rot }}$. Each of these times is defined as the time for characteristic variation of the interaction potential with changing $R$ ( $\gamma$ fixed) or with changing $\gamma(R$ fixed), respectively. Besides, there are two other characteristic times, the period of rotation of $\mathrm{BC}, T_{\mathrm{BC}}$, and the period of the overall rotation $T_{\mathrm{A}-\mathrm{BC}}$. Due to the short range of the interaction responsible for the vibrational transition, the encounter times $\tau_{\text {tr }}$ and $\tau_{\text {rot }}$ are considerably shorter than $T_{\mathrm{A}-\mathrm{BC}}$. This implies that during the vibrationally-inelastic event the collision axis rotates over a small angle and, therefore, the Coriolis interaction can be neglected for a period of the order of the encounter times. This approximation allows one to consider $\varphi$ as an ignorable (i.e. cyclic) variable and regard its conjugate momentum $m$, the projection of the angular momentum of the rotor onto the collision axis, as a constant. The quantum version of this approximation is illustrated by the reasonably good performance of the CS approximation, which ignores the Coriolis coupling in the scattering equations in the $J, j, m$ representation, compared to the accurate $\mathrm{CC}$ approach.

Once the Coriolis coupling is neglected and the classical Hamiltonian is parameterized by the conserved quantity $m$, the two variables $R$ and $\gamma$ can be further transformed into the variables $Q$ and $q$ in order to include the major part of the interaction with the vibration into the $Q-r$ coupling and minimize the interaction within the $q-r$ coupling. If the latter is neglected completely, the $q$ variable becomes a spectator mode, and $Q$ becomes the driving mode. The choice of an optimal transformation $R, \gamma \rightarrow Q, q$ depends on the relation between the encounter times, $\tau_{\text {tr }}$ and $\tau_{\text {rot }}$.

If $\tau_{\mathrm{tr}} \ll \tau_{\text {rot }}$, the rotation of BC only weakly affects the vibrational relaxation. In the limit, the diatom does not rotate at all (in the BF frame) and the anisotropy of the interaction shows up only in the parametric dependence of the transition probability on the orientation angle $\gamma$ (IOS model). A special case of this model corresponds to an isotropic interaction (the breathing sphere, BS, model).

If $\tau_{\text {tr }}$ is of the order of $\tau_{\text {rot }}$, translation and rotation should be treated on the same footing. This treatment is restricted by the condition that the range of the angular motion important for the vibrational relaxation dynamics should be substantially smaller than the angular range of the anisotropy (the effective mass, EM, model). This condition does not prevent the passage to the limit $\tau_{\text {tr }} \ll \tau_{\text {rot }}$, implying that the IOS model is a special case of the EM model.

If $\tau_{\text {tr }} \gg \tau_{\text {rot }}$, the vibrational relaxation occurs mainly as the result of energy transfer from the vibrational to the rotational mode of the A-BC complex at a fixed (but subject to averaging) interfragment distance $R$ (the hindered rotor, $\mathrm{HR}$, model). The two versions of this model are the static $\left(\tau_{\mathrm{tr}} \ll\right.$ 
$T_{\mathrm{BC}}$ ) and adiabatic $\left(\tau_{\mathrm{tr}} \gg T_{\mathrm{BC}}\right.$ ) one. In the following, we will discuss the semiclassical versions of the IOS, EM, and HR models in connection with the mechanisms of the vibration-totranslation, vibration-to-rotation-translation and vibrationto-rotation energy transfer.

\subsection{Vibrational to translational energy transfer. Breathing sphere and infinite-order-sudden models}

The first three-dimensional (3-D) generalization of the collinear LT model was suggested by Takayanagi ${ }^{6}$ and by Schwartz and Herzfeld ${ }^{7}$ in the framework of the breathing sphere (BS) model. This model is based on the assumption that both the vibrationally unperturbed potential $U_{\mathrm{BS}}(R)$ and the vibrational perturbation are spherically symmetric. Since in this case, the 3-D relative motion (collisions with different relative angular momenta) reduces to $1 \mathrm{D}$ motion in the field of the effective potential, one can easily generalize the collinear LT model to three dimensions. In particular, the encounter time will depend on the relative angular momentum $L$ as:

$$
\tau^{3-\mathrm{D} \mathrm{BS}}\left(E_{t}, L\right)=\operatorname{Im}\left\{\int_{R_{s}}^{R_{\mathrm{t}}} \frac{\mathrm{d} R}{\sqrt{(2 / \mu)\left(E_{\mathrm{t}}-U_{\mathrm{BS}}(R)-L^{2} / 2 \mu R^{2}\right)}}\right\}
$$

The major simplification in calculating $\tau^{3-\mathrm{D} \text { BS }}$ comes from the fact that the centrifugal potential varies much slower with $R$ than $U_{\mathrm{BS}}(R)$, such that the former can be considered as a constant with $R=R^{\neq}$where $R=R^{\neq}$is defined through the head-on collision $\left.E_{\mathrm{t}}=U_{\mathrm{BS}}\left(R^{\neq}\right)\right)^{6,7}$ Therefore, one writes

$$
\begin{aligned}
\tau^{3-\mathrm{D} \mathrm{BS}}\left(E_{\mathrm{tr}}, L\right) & \approx \tau^{1-\mathrm{D} \mathrm{BS}}\left(E_{\mathrm{R}}\right) \\
& =\operatorname{Im}\left\{\int_{R_{\mathrm{S}}}^{R_{\mathrm{t}}} \frac{\mathrm{d} R}{\sqrt{(2 / \mu)\left(E_{\mathrm{R}}-U_{\mathrm{BS}}(R)\right)}}\right\}
\end{aligned}
$$

Here $E_{\mathrm{R}}=E_{\mathrm{t}}-L^{2} / 2 \mu\left(R^{\neq}\right)^{2}$ and $\tau^{1 \mathrm{D}-\mathrm{BS}}\left(E_{\mathrm{R}}\right)$ is the $1 \mathrm{D}$ encounter time that depends on the radial energy, $E_{\mathrm{R}}=E_{\mathrm{t}}$ $-L^{2} / 2 \mu\left(R^{\neq}\right)^{2}$. Now, similar to the original LT model and its CCT and SC generalisations, one calculates BS-CT, BS-CCT and BS-SC transition probabilities and their average counterparts $\bar{P}_{n n^{\prime}}{ }^{1 \mathrm{D}-\mathrm{BS}(T)}$. When one takes into account the strong dependence of the transition probability on the encounter time, the 3-D BS rate coefficient, which is obtained by integration of the mean 1-D transition probability over all possible values of $L$, is expressed as

$$
k_{n n^{\prime}}^{\mathrm{BS}}(T)=\pi\left(R^{\neq}\right)^{2} \bar{\nu}^{3-\mathrm{D}} \times \bar{P}_{n n^{\prime}}^{1-\mathrm{D} \mathrm{BS}}(T)
$$

where $\bar{\nu}^{3-\mathrm{D}}=\sqrt{8 k_{\mathrm{B}} T / \pi \mu}$ is the mean $3-\mathrm{D}$ relative velocity. The first factor at the r.h.s. of eqn (5.3) is close to the gaskinetic collision number, and the second is the mean transition probability for collinear collisions within the BS potential

$$
\bar{P}_{n n^{\prime}}^{1-\mathrm{D} \mathrm{BS}}(T)=\int_{0}^{\infty} P_{n n^{\prime}}^{1-\mathrm{D} \mathrm{BS}}\left(E_{\mathrm{i}}\right) \exp \left(-E_{\mathrm{i}} / k_{\mathrm{B}} T\right)\left(\mathrm{d} E_{\mathrm{i}} / k_{\mathrm{B}} T\right)
$$

Expressions (5.3) and (5.4) for $k_{n n^{\prime}}{ }^{\mathrm{BS}}$ may also be interpreted in the language of transition state theory which represents the state-to-state rate coefficient $n \rightarrow n^{\prime}$ as the product of a mean
1-D state-to-state probability density flux $\bar{v}^{1 \mathrm{D}} \bar{P}_{n n^{\prime}}{ }^{1-\mathrm{D}} \mathrm{BS}(T)$ (with $\bar{v}^{1 \mathrm{D}}$ equal to the $1-\mathrm{D}$ mean relative velocity, $\left.\bar{\nu}^{1-\mathrm{D}}=\sqrt{k_{\mathrm{B}} T / 2 \pi \mu}\right)$ and the total area of the dividing surface $4 \pi\left(R^{\neq}\right)^{2}$. The vibrational transition is assumed to occur with the probability $\bar{P}_{n n^{\prime}}{ }^{1 \mathrm{D}-\mathrm{BS}}$ each time when a trajectory crosses normally the dividing surface, i.e. the sphere of radius $R^{\neq}$. An additional correction should be introduced into the pre-exponential factor of the BS relaxation rate, which accounts for a possible dependence of the perturbation on the angle $\gamma$ between the collision axis $\boldsymbol{r}$ (the vector joining the atom and the centre of mass of the diatomic) and the molecular axis $\boldsymbol{r}$ but which does not affect the temperature dependence of the rate. ${ }^{7}$

For the $1 \rightarrow 0$ vibrational transition, the explicit temperature dependence of $k_{10}^{\mathrm{BS}}$ in the CTC approximation with the LT encounter time from eqn (3.6), reads: ${ }^{7}$

$$
\begin{aligned}
& k_{10}^{\mathrm{BS}-\mathrm{CTC}} \propto T^{1 / 3} \exp \left(-3\left(\frac{T_{\mathrm{BS}}}{T}\right)^{1 / 3}+\frac{T_{\mathrm{vib}}}{2 T}\right) \\
& T_{\mathrm{BS}}=\pi^{2} \omega^{2} \mu a^{2} / 2 k_{\mathrm{B}}
\end{aligned}
$$

where $T_{\mathrm{BS}}$ coincides with $T_{\mathrm{LT}}$ from eqn (3.8), and $T_{\mathrm{vib}}=$ $\hbar \omega / k_{\mathrm{B}}$. The pre-exponential factor $T^{1 / 3}$ results from multiplying $T^{1 / 2}$ (arising from the temperature dependence of the collision number) and $T^{-1 / 6}$ (arising from the SD calculation of the integral in eqn (5.4)). The temperature dependence of this factor is too weak to be discerned in the LT plots of the rate coefficient, $\ln k_{10}^{\mathrm{BS}}(T)$ vs. $T^{-1 / 3}$. We therefore conclude that, within the CTC approach, the curvatures of LT plots can only be due to deviations of the potential from the exponential form and to the first quantum correction, eqn (5.5).

This form of $k_{10}^{\mathrm{BS}-\mathrm{CCT}}(T)$ has widely been used in the interpretation of experimental results on the vibrational relaxation of symmetric (homonuclear, as $\mathrm{N}_{2}$ ) and slightly-asymmetric (as CO) molecules at high and medium temperatures. For these molecules, the noticeable curvature of the LT plots (below, say, $500 \mathrm{~K}$ ) was ascribed to the effect of the term $T_{\mathrm{vib}} / 2 T$ in eqn (5.5). ${ }^{76}$ The SC counterpart of eqn (5.5) is obtained from eqn (4.3)) with the LT encounter time $\tau(E)=\pi a / \sqrt{2 E / \mu}$. The expression for $k_{10}^{\mathrm{BS}-\mathrm{CCT}}$ includes the same parameters as eqn (5.5); however, $k_{10}^{\mathrm{BS}-\mathrm{SC}}$ yields a more general description than $k_{10}^{\mathrm{BS}-\mathrm{CTC}}$, being valid at lower temperatures for the same type (exponential) repulsive interaction. Fig. 2 compares the dependences of $k_{10}^{\mathrm{BS}-\mathrm{CT}}, k_{10}^{\mathrm{BS}-\mathrm{CTC}}$ and $k_{10}^{\mathrm{BS}-\mathrm{SC}}$ on $\left(T / T_{\mathrm{BS}}\right)^{-1 / 3}$ for a value of $T_{\mathrm{vib}} / T_{\mathrm{BS}}$ which is appropriate for the $\mathrm{N}_{2}-\mathrm{He}$ collisions. ${ }^{77}$ One sees how the incipient curvatures of the $\mathrm{SC}$ results are reproduced by the CCT results in the temperature range shown. We emphasize that eqn (5.5) and its CS counterpart are only appropriate for the description of the rate coefficient for a repulsive exponential potential, i.e. for a potential with a constant steepness such as characterized by its logarithmic derivative. At high temperatures this approximation is usually satisfactory, but at medium temperatures due to the influence of the attractive part of the potential it breaks down.

A simple generalization of the BS model is the infinite order sudden (IOS) model. The dividing surface is now an axiallysymmetric distorted sphere specified by the equation $R^{\neq}=$ $R^{\neq}(\gamma)$. The radial motion is again considered to be the mode 


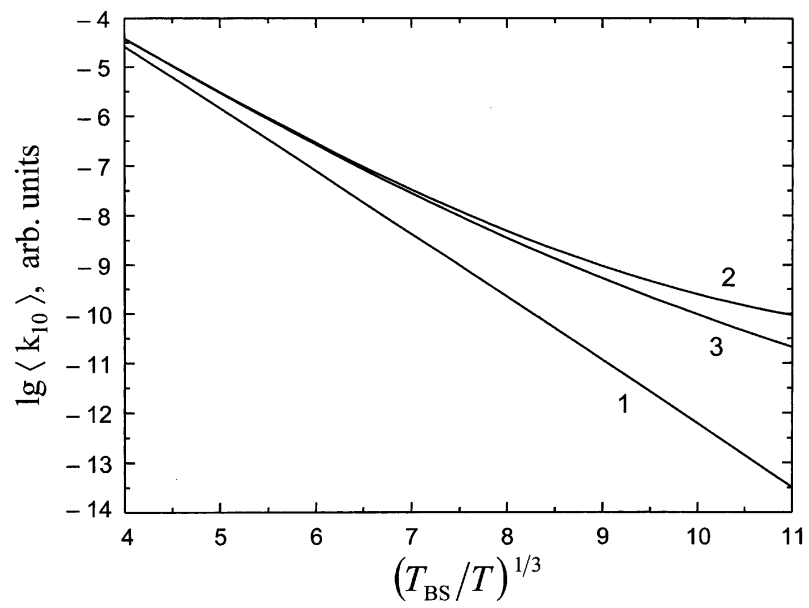

Fig. 2 Comparison of CT (curve 1), CCT (curve 2) and SC (curve 3) representations of BS rate coefficients $k_{10} v s$. the $\mathrm{LT}$ variable $\left(T_{\mathrm{BS}} / T\right)^{1 / 3}$ (for exponential repulsive interaction with the parameter $\left(T_{\mathrm{BS}} / T_{\mathrm{vib}}\right)$ appropriate for $\mathrm{N}_{2}+\mathrm{He}$ collisions).

that induces the vibrational transition, while the potential that drives this mode $U_{\operatorname{IOS}}(\mathrm{R} ; \gamma)$ is the original potential $U(R, \gamma)$ in which $\gamma$ is regarded as a parameter. Thus, the rotation of the molecule plays the part of a spectator that affects the $R$-dependent potential but does not participate as a dynamical mode. Therefore, the appropriate encounter time $\tau^{1-\mathrm{D}}$ IOS, beside $E_{\mathrm{R}}$, also depends on $\gamma$, such that the following relations follow:

$$
\bar{P}_{n n^{\prime}}^{1-\mathrm{D} \operatorname{IOS}}(T, \gamma)=\int_{0}^{\infty} P_{n n^{\prime}}^{1-\mathrm{D} \operatorname{IOS}}\left(E_{R}, \gamma\right) \exp \left(-E_{R} / k_{\mathrm{B}} T\right)\left(\mathrm{d} E_{R} / k_{\mathrm{B}} T\right)
$$

$$
k_{n n^{\prime}}^{\mathrm{IOS}}(T)=2 \pi \bar{v}^{1 D} \int_{0}^{\pi} \bar{P}_{n n^{\prime}}^{1-\mathrm{D} \operatorname{IOS}}(T, \gamma)\left(R^{\neq}(\gamma)\right)^{2} \sin \gamma \mathrm{d} \gamma
$$

For a dividing surface $\mathrm{S}^{\neq}$, whose element $\mathrm{d} S^{\neq}=$ $2 \pi\left(R^{=}(\gamma)\right) \sin \gamma \mathrm{d} \gamma$ is not very different from that of a sphere, the temperature dependence of $k_{n n^{\prime}}^{\text {IOS }}$ is then virtually the same as that of $k_{n n^{\prime}}$ BS

\subsection{Vibrational to translational and rotational energy transfer: the effective mass model}

The effective mass (EM) approach for calculating transition probabilities was formulated in ref. 78 and 79 as a generalization of the model proposed by Moore. ${ }^{80}$ In the latter model, the LT encounter time was modified in such a way that the translational relative energy $E_{\mathrm{t}}$ was replaced by the rotational energy $E_{\mathrm{r}}$ of the diatom and the reduced mass $\mu$ was replaced by the ratio $I / d^{2}$ where $d$ is the distance between the centre of mass of $\mathrm{BC}$ and the peripheral atom. In this way the vibrational transition was considered to be induced by the rotation of $\mathrm{BC}$, with rebound character of the rotational trajectory similar to that of the translational trajectory of the LT model. Within this approach, the length parameter $a$ in the original LT expression, was used as a purely empirical quantity.
The more sophisticated EM model, also called the breathing shell model to emphasize its difference from the breathing sphere model, is described in detail in ref. 9, and it was refined in later articles. ${ }^{81-87}$ The EM approximation for the determination of common trajectories consists in the introduction of a single "driving mode" $Q$ in a localized region of the $R, \gamma$-space where the vibrational transition occurs. The driving mode is a specific combination of the coordinate displacements $\Delta R$ and $\Delta \gamma$ that describes the motion along the gradient of the potential $U(R ; \gamma)$ in a small region centred at $R^{*}, \gamma^{*}$. The 2-D potential $U\left(R^{*}+\Delta R ; \gamma^{*}+\Delta \gamma\right)$ is then replaced by a 1-D potential $U^{\mathrm{EM}}\left(Q ; R^{*}, \gamma^{*}\right)$ "along the gradient" and the reduced mass $\mu$ is changed into an effective mass $\mu^{*}$ which depends on $\mu$, the moment of inertia of the diatom $I$, and the contour line $R^{*}=$ $R^{*}\left(\gamma^{*}, E\right)$ of the potential $U\left(R^{*}, \gamma^{*}\right)$ for a given total energy, i.e. the function $R^{*}=R^{*}\left(\gamma^{*}, E\right)$ is found as a solution of the equation $U\left(R^{*}, \gamma^{*}\right)=E$. Thus, the applicability of the EM approach in accounting for the effect of rotation of the diatom onto vibrational relaxation is limited by the condition that the rotation angle of the diatom axis with respect to the collision axis during the encounter time should be small compared to the angular range of the interaction anisotropy (implying the relation $\left|\mathrm{d} R^{*} / \mathrm{d} \gamma^{*}\right| \ll R^{*}$ ) and that the anisotropy of the effective mass is not very large (see below). Under this condition the expression for the effective mass $\mu^{*}$ assumes the form

$$
\mu^{*}\left(\gamma^{*}, E\right)=\mu /\left(1+\mu\left(\mathrm{d} R^{*} / \mathrm{d} \gamma^{*}\right)^{2} / I\right)
$$

A simple expression for the effective mass for a homonuclear diatom, adopted in ref. 77, corresponds to a contour line

$$
R^{*}\left(\gamma^{*}, E\right)=R_{0}(E)-\Delta R_{0}(E) \cos ^{2} \gamma^{*}
$$

for which

$$
\mu_{*}=\mu I /\left(I+\mu \Delta R_{0}^{2}(E) \sin ^{2} 2 \gamma^{*}\right)
$$

A useful approximation for the effective mass was suggested in ref. 86 for the case when the interaction potential $U(R, \gamma)$ can be represented, for a certain geometry of the A + BC system, as a function of the distance $R_{\mathrm{XC}}$ between the atom $\mathrm{C}$ and the atom $\mathrm{X}(\mathrm{X}=\mathrm{A}$ or $\mathrm{B})$ of the diatom $\mathrm{BC}$. In this case, the expression for $\mu^{*} \equiv \mu_{\mathrm{X}}^{*}$ reads

$$
\mu_{\mathrm{X}}^{*}=\mu I /\left(I+\mu r_{\mathrm{X}}^{2} \sin ^{2} \theta^{*}\right)
$$

where $r_{\mathrm{X}}$ is the distance from the centre of mass of $\mathrm{BC}$ to atom $\mathrm{X}(\mathrm{X}=\mathrm{B}$ or $\mathrm{C})$, and $\theta^{*}$ is the angle between the vectors $\boldsymbol{r}_{\mathrm{AX}}$ and $\boldsymbol{r}_{\mathrm{BC}}$. The dependence of $\theta^{*}$ on $\gamma^{*}$ and $E$, which appears as the $\gamma^{*}, E$ dependence of the effective mass can be found from simple geometrical considerations. In ref. 87, a more general expression for $\mu_{\mathrm{X}}^{*}$, still formally retaining the form of eqn (5.11) but with modified meaning of $r_{\mathrm{X}}$ and $\theta^{*}$, was suggested. Detailed discussions of the angular dependence of the effective mass can be found in ref. 86 for $\mathrm{Li}_{2}-$ noble gas and in ref. 87 for $\mathrm{HCl}-\mathrm{Ar}$ systems. Qualitatively, the angular dependence of the EM can be characterised by the ratio of the minimum of the EM, $\mu^{\dagger}=\min \left\{\mu^{*}\right\}$, to the reduced mass $\mu$ of the collision partners, $\mu^{\dagger} / \mu$. For instance, the values of this ratio were found to be about 0.9 for $\mathrm{N}_{2}-\mathrm{He},{ }^{77} 0.7$ for $\mathrm{Li}_{2}-\mathrm{Ar}^{86}$ and 0.37 for $\mathrm{HCl}-\mathrm{Ar}{ }^{87}$ 
Though, in principle, $\mu^{*}$ depends on the energy and the angle, the former dependence can often be neglected due to the weak dependence of the contour lines on the energy. Then $\mu^{*}$ and $U^{\mathrm{EM}}$ can be parameterized only through $\gamma^{*}$ (i.e. $\mu^{*}=$ $\mu^{*}\left(\gamma^{*}\right)$ and $\left.U^{\mathrm{EM}}=U^{\mathrm{EM}}\left(q, \gamma^{*}\right)\right)$, such that the EM encounter time $\tau_{\mathrm{EM}}$ and 1-D CT transition probability also depend on $\gamma^{*}$ :

$$
P_{10}^{\mathrm{EM}-\mathrm{CT}}\left(E_{\mathrm{Q}} ; \gamma^{*}\right)=A_{10}^{\mathrm{EM}-\mathrm{CT}}\left(E_{\mathrm{Q}} ; \gamma^{*}\right) \exp \left(-2 \omega \tau_{\mathrm{EM}}\left(E_{\mathrm{Q}}, \gamma^{*}\right)\right)
$$

The 3-D EM rate coefficient is obtained from the 1D counterpart as a result of integration of the mean probability $\bar{P}_{n n !}^{1-\mathrm{D} \text { EM }}\left(T, \gamma^{*}\right)$ over all points on the surface $S^{\neq}$:

$$
k_{n n^{\prime}}^{\mathrm{EM}-\mathrm{CT}}(T)=2 \pi \int_{0}^{\pi} \sqrt{\frac{k_{\mathrm{B}} T}{2 \pi \mu^{*}}} \bar{P}_{n n^{\prime}}^{\mathrm{EM}-\mathrm{CT}}\left(T, \gamma^{*}\right)\left(R^{*}\left(\gamma^{*}\right)\right)^{2} \sin \gamma^{*} \mathrm{~d} \gamma^{*}
$$

The contribution of $\bar{P}_{n n^{\prime}}{ }^{\text {EM-CT }}$ to the integral in eqn (5.18) is determined by the interplay of two $\gamma^{*}$-dependent factors, the exponential and pre-exponential. The $\gamma^{*}$ dependence of the former is determined by vibrationally-elastic scattering, while that of the latter arises from the coupling interaction. Such an interplay is shown in Fig. 4 of ref. 86 for $\mathrm{Li}_{2}$-noble gas collisions: though the effective mass decreases monotonically within the range of $0<\theta^{*}<\pi / 2$ leading to a monotonic increase of the exponential, the probabilities, due to the decrease of the coupling strength with $\theta^{*}$, attain their maximum at about $\theta^{*}=\pi / 4$. This behavior of the probability makes it difficult to predict the temperature dependence of the rate constant on the basis of pure vibrationally-elastic scattering, as was done in the BS model. However, if the function $\mu^{*}$ $=\mu^{*}\left(\gamma^{*}\right)$ possesses a noticeable minimum at $\gamma^{*}=\gamma^{\dagger}$, such that $\mu^{*}\left(\gamma^{\dagger}\right)=\mu^{\dagger}$, the integral in eqn (5.13) is dominated by a small range of $\gamma^{*}$ values near $\gamma^{\dagger}$ that determines an optimal collision configuration with a 1-D transition probability. This permits one to derive the temperature dependence of the rate coefficients in an optimal effective mass (OEM) approximation. For instance, within the CTC approach and a repulsive exponential potential, we get

$$
\begin{aligned}
& k_{10}^{\mathrm{OEM}-\mathrm{CCT}}(T)=T^{1 / 2} \exp \left(-3\left(\frac{T_{\mathrm{EM}}^{\dagger}}{T}\right)^{1 / 3}+\frac{T_{\mathrm{vib}}}{2 T}\right) \\
& T_{\mathrm{EM}}^{\dagger}=\pi^{2} \omega^{2} \mu^{\dagger} a^{2} / 2 k_{\mathrm{B}}
\end{aligned}
$$

Different from the BS model, the pre-exponential factor in eqn (5.14) is now proportional to $T^{1 / 2}$ : besides the $T^{1 / 3}$ dependence of the BS model (see comment to eqn (5.5)) it includes an additional factor proportional to $T^{1 / 6}$. The latter reflects the growing contribution (with increasing temperature) of collision configurations with $\gamma^{*}$ close to $\gamma^{\dagger}$ (i.e. an opening of the acceptance cone for effective collisions). Eqn (5.14) allows one to find corrections to the BS approximation since the latter is a special case of the EM approximation. A proper parameter that describes the contribution of the rotation to the "alongthe-gradient" mode for the elliptic equipotential surface in eqn (5.9) is $Q=\mu(\Delta R)^{2} / I^{88}$ which is the same parameter as suggested for the characterization of rotational-to-translational energy exchange in ref. 89.

The given CCT rate coefficients can be easily reformulated in the SC approximation since this generalization basically repeats that for the BS or IOS models (section 4). In section 6 this is exemplified for the relaxation of $\mathrm{N}_{2}$ in He. A simplified version of the SC generalization, based on the symmetrization of velocities (see eqn (4.12)) within the exponential potential, was used in ref. 87 for the $\mathrm{HCl}+\mathrm{Ar}$ system.

In the case of vibrational deactivation, the EM model predicts a release of the energy in the form of kinetic energy with the linear momentum $p_{q}$ in the direction of the gradient of the PES, with the normal (to the plane A-BC in the encounter configuration) component $j_{q}$ of the angular momentum of the diatom changing its sign as a result of the encounter. Upon receding of the partners, two dynamical quantities, $p_{q}$ and $j_{q}$, combine with other dynamical quantities that played the part of spectators, to produce asymptotically two conserved vector quantities, the linear momentum of the relative motion $\boldsymbol{p}$ and the angular momentum of the diatom $\boldsymbol{j}$. This yields, in principle, the distribution of the released energy between the relative translation and intrinsic rotation, though the determination of this distribution is beyond the scope of the EM model.

The trajectory calculations confirmed the above features of the EM model for not too high values of $j$, but also indicated yet another outcome of the encounter, for high values of $j$, in which the component $j_{q}$ does not change its sign. ${ }^{86}$ These two types of encounters, studied for planar collisions, were dubbed ordinary and extraordinary ${ }^{86}$ with the latter producing stronger rotational excitation. In fact, the appearance of extraordinary encounters is an indication that the rotational motion becomes more effective in producing vibrational deactivation than the along-the-gradient motion. The limiting situation, when the latter is considered totally ineffective, is described by the hindered rotor model discussed in the next section.

\subsection{Vibrational to rotational energy transfer. Hindered rotor model}

The hindered rotor model (HR) was suggested by Ovchinnikova $^{75}$ for the description of the vibrational relaxation under the condition $\tau_{\mathrm{tr}} \gg \tau_{\mathrm{rot}}$. The main idea of this approach is that the vibrational energy of the diatom is transformed mainly into rotational energy, with translational energy being the mode that drives the partners into the interaction region. The HR model is a generalization of a free-rotor model; the latter is based on the observation that the weight of the high-frequency Fourier components of the perturbing force increases rapidly with the decrease in the encounter time which can be associated with fast and, therefore, almost free rotation. Accepting this idea, one calculates the vibrational relaxation rate coefficient as the rotationally-induced vibrational transition probability per one rotation, multiplied by the frequency of rotation and averaged over a Boltzmann distribution of rotational energies. This concept was the basis for several treatments..$^{90-94}$

The HR model ${ }^{75,95}$ takes into account a hindered rotation of the diatom. Within this approach one can consider two limiting possibilities that differ by the ratio of the translational 
encounter time $\tau_{\operatorname{tr}}$ of the $\mathrm{A}-\mathrm{BC}$ complex and the period of rotation of BC, $T_{\mathrm{BC}}$. Under the condition $\tau_{\text {rot }} \ll \tau_{\mathrm{tr}} \ll T_{\mathrm{BC}}$, the vibrational relaxation occurs as a result of a single rotational encounter during the $\mathrm{A}+\mathrm{BC}$ collision while, with the condition $\tau_{\text {rot }} \ll T_{\mathrm{BC}} \ll \tau_{\text {tr }}$, it occurs as the result of multiple rotational encounters during the $\mathrm{A}+\mathrm{BC}$ collision. It turned out that these two possibilities, called the static and adiabatic mechanisms, respectively, do not differ much in the temperature dependence of the vibrational relaxation rate, so that we will not discriminate them in the following. The main idea of the HR model is that the evolution of the rotational mode is determined by a strongly anisotropic potential $U(R, \gamma)$ at fixed values of $R$. The trajectories of the rotational mode and the high-frequency Fourier components of the perturbation, needed for the implementation of the CT and CCT approximation into the calculation of vibrational transition probabilities at fixed values of $R$, were found numerically, and the rate coefficient was calculated by an appropriate averaging of the $R$-dependent transition probabilities.

Considerable insight into the energy-transfer mechanism within the HR model is gained by using a model potential of the form ${ }^{96}$

$$
U_{\mathrm{HR}}^{\text {aniso }}(R, \gamma)=U_{1} \cosh ^{-2}\left(\gamma / 2 \gamma_{0}\right)
$$

Two functions of $R$, the prefactor $U_{1}$ that yields the height of the rotational barrier at the linear configuration at $\gamma=0$ and the angular scale parameter $\gamma_{0}$ that characterises the sharpness of the anisotropic interaction, should be determined by fitting $U_{\mathrm{HR}}^{\text {aniso }}$ to the anisotropic part of an ab initio potential in the region where the rotational encounter is deemed to occur. We can see that for $\gamma_{0} \ll 1$ the barrier is quite sharp and for $\gamma / \gamma_{0} \gg$ 1 the angular dependence of $U_{\mathrm{HR}}^{\text {aniso }}$ is exponential, similar to the potential in the LT model. An interesting feature of the potential (5.15) is that the encounter time is quite insensitive to the height of the rotational barrier, and for a planar motion it does not depend on $U_{1}$ at all

$$
\begin{aligned}
\tau_{\mathrm{HR}}\left(E_{\gamma}\right) & =\operatorname{Im}\left\{\int_{\gamma_{\mathrm{s}}}^{\gamma_{\mathrm{t}}} \frac{\mathrm{d} \gamma}{\sqrt{(2 / I)\left(E_{\gamma}-U_{\mathrm{HR}}^{\text {aniso }}(R, \gamma)\right)}}\right\} \\
& =\pi \gamma_{0} \sqrt{\frac{I}{2 E_{\gamma}}}
\end{aligned}
$$

Here $E_{\gamma}$ is the energy of the $\gamma$ mode, and $\gamma_{\mathrm{s}}$ is a singular point of the vibrational-rotational coupling potential. In distinction to the LT, SSH, IOS and EM models, where the driving-mode trajectory corresponds to a rebound motion, the trajectories for the HR model show either rebound or glancing character depending on the relation between $E_{\gamma}$ and the barrier height $U_{1}(R)$. Accordingly, the turning point angle $\gamma_{\mathrm{t}}$ is real for $E_{\gamma}<U_{1}(R)$ and imaginary for $E_{\gamma}>U_{1}(R)$ while $\gamma_{\mathrm{s}}$ is always imaginary. However, in both cases the HR encounter time $\tau_{\mathrm{HR}}\left(E_{\gamma}\right)$ for the HR model potential in eqn (5.16) is given by the same expression. It was shown that the vibrational transition probability decreases rapidly with increasing $m$, such that the temperature dependence of the relaxation rate averaged over $m$ is close to that for the planar collision. ${ }^{97}$ Thus, on the CT level of approximation, the transition probability per one rotational encounter assumes the form

$$
P_{10}^{\mathrm{HR}-\mathrm{CT}}\left(E_{\gamma} ; R\right)=A_{10}^{\mathrm{HR}-\mathrm{CT}}\left(E_{\gamma} ; R\right) \exp \left(-2 \omega \tau_{\mathrm{HR}}\left(E_{\gamma}\right)\right)
$$

In this expression, the exponential factor assumes the same form for rebound and glancing encounters and the difference appears in the pre-exponential factor only. When averaged over a thermal distribution of rotational energies, eqn (5.17) determines the mean transition probability $\bar{P}_{10}^{\mathrm{HR}-\mathrm{CT}}(T, R)$ which, in turn, enters the expression for the HR vibrational relaxation rate coefficient:

$$
k_{10}^{\mathrm{HR}-\mathrm{CT}}(T)=4 \pi \int_{0}^{\infty} \nu_{r}(R) \bar{P}_{10}^{\mathrm{HR}-\mathrm{CT}}(T, R) F(R, T) R^{2} \mathrm{~d} R
$$

where $\nu_{\gamma}(R)$ is the frequency of visiting the encounter region by the hindered rotor mode at fixed $R$, and the integration over $R$ with a Boltzmann distribution function $F(R, T)$ represents the averaging over all possible separations between the collision partners. Note that the averaging over the separations in eqn (5.18) for the HR model is analogous to the averaging over the angles in eqn (5.13) for the EM model. In a similar way, a CCT version of the relaxation rate can be obtained, as well as its $\mathrm{SC}$ counterpart. $^{98}$

Since the dependence of $\tau_{\mathrm{HR}}$ on the energy of the hindered rotation is the same as that of the LT encounter time on the translational energy, one can expect a similar temperature dependence of the relaxation rate. In particular, $k_{10}^{\mathrm{HR}-\mathrm{CCT}}$ assumes the form

$$
\begin{aligned}
& k_{10}^{\mathrm{HR}-\mathrm{CCT}}(T) \propto \exp \left(-3\left(\frac{T_{\mathrm{HR}}}{T}\right)^{1 / 3}+\frac{T_{\mathrm{vib}}}{2 T}\right) \\
& T_{\mathrm{HR}}=\pi^{2} \omega^{2} I \gamma_{0}^{2} / 2 k_{\mathrm{B}}
\end{aligned}
$$

However, the temperature dependence of the pre-exponential factor in eqn (5.19) is somewhat more complicated compared to the EM-CTC version which is due to the presence of a nonLT pre-exponential factor in the expression for $\bar{P}_{10}^{\mathrm{HR}-\mathrm{CTC}}(T, R)$ and to the averaging over the separations affected both by the isotropic and anisotropic parts of the interaction potential. Fitting the parameters of the potential from eqn (5.15) to $a b$ initio or semiempirical potentials, the CCT version of the HR model was used for the interpretation of experimental data on the vibrational relaxation of hydrogen halide molecules. ${ }^{99}$

\section{Temperature dependence of vibrational relaxation rates within the EM and HR models}

This section presents specific examples that illustrate the performance of generalized LT models, describing the temperature dependence of vibrational relaxation rate coefficients and identifying the driving mode that induces the vibrational transition.

Our first example is the relaxation of $\mathrm{N}_{2}$ in a He heat bath. For this system, there exist accurate quantum calculations of the rate coefficients within the CC and CS approaches, and experimental results are available over a wide temperature range. An estimation of the ratio $\tau_{\mathrm{tr}} / \tau_{\text {rot }}$, which turns out to be 
smaller than unity, indicates that this system falls into the category covered by the EM model. It thus becomes possible to compare the temperature dependence predicted by the SC EM model with that from the quantum calculations and from experimental data.

Our second example is the relaxation of HF in an Ar heat bath. For this process, the ratio $\tau_{\mathrm{tr}} / \tau_{\text {rot }}$ is larger than unity which suggests the applicability of the HR model. The HR calculations of the rate coefficients, performed quite some time ago, can be compared with recent accurate quantum results. However, a comparison with experimental data is not unproblematic since these may not represent state-to-state rate coefficients (see below for details) though the results of the HR calculations reasonably well agree with the experimental data for some PES (and disagree for others).

Our third example is the relaxation of $\mathrm{HCl}$ in Ar. Here we compare the vibrational relaxation times calculated for different choices of the driving modes but with the same model PES. This comparison demonstrates big differences between relaxation rates determined within the infinite-order sudden approach, effective mass model, and the hindered rotor model. It also shows the satisfactory performance of the analytical HR model in describing the temperature dependence of the relaxation rate.

\subsection{Relaxation of $\mathrm{N}_{2}$ in He: weak effect of rotation}

The vibrational relaxation of $\mathrm{N}_{2}$ in a He heat bath

$$
\mathrm{N}_{2}(v=1)+\mathrm{He} \rightarrow \mathrm{N}_{2}(v=0)+\mathrm{He}
$$

can serve as a simple illustration of the application of the EM $\mathrm{SC}$ method described in section 3 . We have chosen this system for three reasons:

(i) The low anisotropy of the interaction, the small reduced mass of the partners and the rather high value of the moment of inertia of $\mathrm{N}_{2}$ result in an effective mass that deviates only little from $\mu$. This suggests the use of the EM model which is also expected not to differ too much from the BS model.

(ii) There exist accurate quantum calculations ${ }^{100,101}$ which provide the basis for a check of the SC approximation.

(iii) The experimental data, available over a very wide temperature range, from several thousand degrees ${ }^{102}$ down to $70 \mathrm{~K},{ }^{100,101,103}$ are in agreement with accurate quantum calculations and can be compared with the SC theoretical results.

The above features suggest that the temperature dependence of the rate coefficient for the temperatures that are still noticeably higher than the characteristic temperature of the potential well, $T_{\mathrm{W}}=D / k_{\mathrm{B}} T \approx 30 \mathrm{k}$, are well described by the BS-CCT approach. Indeed, the experimental values of $k_{10}(T)^{102,103}$ are reproduced by eqn (5.5) in the range 2500-300 K (with a noticeable deviation from the LT plot at the low-temperature side of this interval) ${ }^{76}$ with a choice $a=$ $0.263 \AA$ corresponding to the older ab initio potential from ref. 104. In the following, we revisit the CCT approximation and discuss the SC approximation, using the more recent ab initio potential and estimating the rotational effect within the EM metod. ${ }^{77}$

The key quantity that determines the temperature dependence of the relaxation rate is the encounter time. It has been calculated for a realistic interaction potential taken from

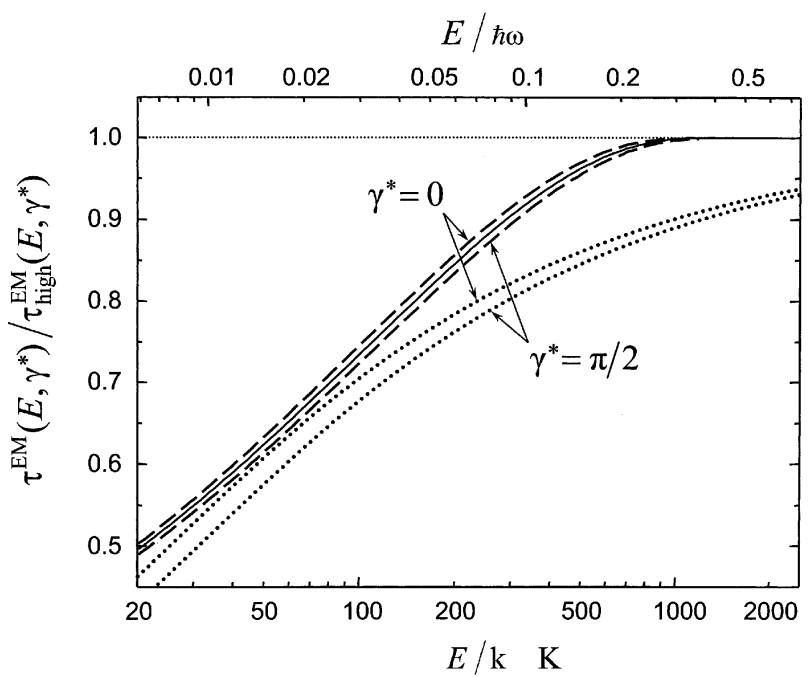

Fig. 3 Relative encounter times for $\mathrm{N}_{2}+$ He collisions for a linear $\left(\gamma^{*}\right.$ $=0)$ and T-shaped $\left(\gamma^{*}=\pi / 2\right)$ geometries $v s$. collision energy. The dashed curves represent the result for an $a b$ initio potential (full line $=$ mean), and dotted curves are for the Morse potential with two ab initio parameters, the high-energy slope and the depth of the potential well.

ref. 100. The interaction potential for high energies, say above $k_{\mathrm{B}} \times 500 \mathrm{~K}$, is close to a purely exponential repulsion with the length parameter $a_{\text {high }}=0.241 \AA$ for the collinear and T-shaped geometry of the collision complex, and this value is close to the independent estimation from the asymptotic method $^{105,106}$ that yields $a_{\text {asym }}=0.244 \AA$. For lower energies, the potential drops more steeply due to the effect of the potential well. This effect is characterised by the deviation of $\tau(E)$ from the high-energy LT encounter time. For the above two geometries, due to symmetry reasons, the effective mass coincides with the reduced mass such that the high-energy encounter time is $\tau_{\text {high }}(E)=\pi a_{\text {high }} / \sqrt{2 E / \mu}$. The ratio $\tau(E) / \tau_{\text {high }}(E)$ is shown in Fig. 3 as a function of the collision energy for two geometries. The difference between the two dashed lines is due to the difference of the well depths in these configurations ( 25 and $31 \mathrm{~K}$, respectively). For a comparison, we also show similar ratios for the Morse potential (see eqn (4.7)) with the same values of $a_{\text {high }}$ and $D$. We can see that the model of a Morse potentials yields quite different encounter times compared to that for the ab initio potentials. This is explained by the fact that the attractive part of the Morse potential, being proportional to $-\exp \left(-R / 2 a_{\text {high }}\right)$, affects the repulsion much stronger than the more realistic attractive interaction being proportional to $-1 / R .^{6}$ The mean transition probabilities for two configurations, $\gamma=0$ and $\gamma=\pi / 2$, have virtually the same temperature dependence because $\tau(E)$ are nearly the same and the effect of rotation does not show up. However, it does for other configurations and is manifested through the dependence of the effective mass $\mu^{*}$ on $\gamma^{*}$. According to eqn (5.10), the minimum value of $\mu^{*}$, called $\mu^{\dagger}$, corresponds to $\gamma^{*}=\pi / 4$, and, with $\Delta R=0.59 \AA$ as appropriate for the $a b$ initio potential, $\mu^{\dagger}$ amounts to about $0.9 \mu$. The only small difference between the effective masses allows one to get useful lower and upper bounds of the EM SC rate coefficient through its IOS-like SC counterparts by calculating IOS SC rate coefficients with angle-independent masses $\bar{\mu}$, 


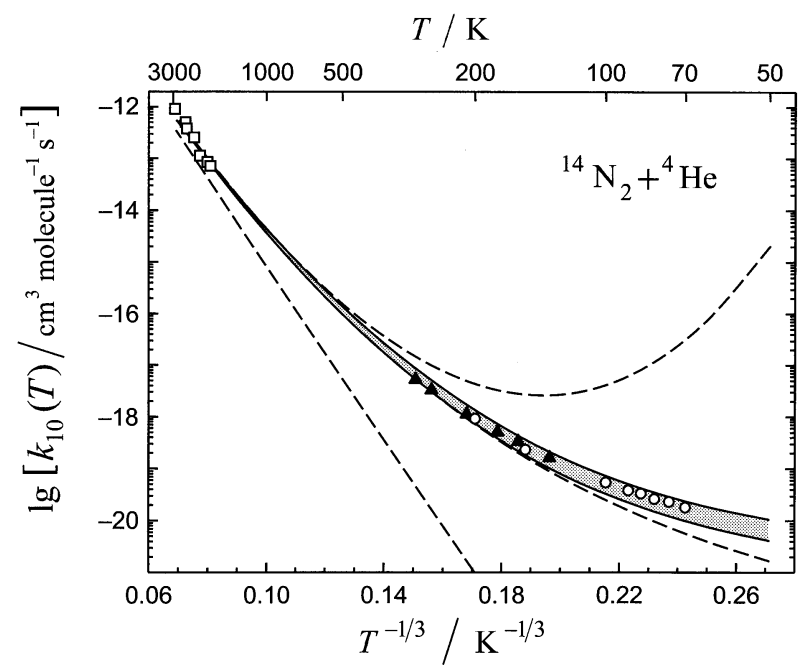

Fig. 4 LT plot of the vibrational relaxation rate coefficient $k_{10}(T)$ for ${ }^{14} \mathrm{~N}_{2}$ in ${ }^{4} \mathrm{He}$ (normalised to high-temperature experimental data from ref. 102). Lower and upper full lines correspond to $\left.k_{10}^{\mathrm{IOS}-\mathrm{SC}}(T)\right|_{\bar{\mu}}=\mu$ and $\left.k_{10}^{\text {IOS-SC }}(T)\right|_{\bar{\mu}}=0.9 \mu$, the shaded area between them accommodates the experimental data (symbols: filled triangles from ref. 103 and open circles from ref. 104) which are essentially in agreement with accurate quantum calculations. The three dashed curves, from bottom to top, represent the rate coefficients calculated in the common trajectory (i.e. Landau-Teller), semiclassical and corrected common trajectory approximations, after ref. 77 .

using the maximal and minimal of their values (that is $\bar{\mu}=\mu$ and $\bar{\mu}=0.9 \mu$, respectively). In this way one obtains

$$
\left.k_{10}^{\mathrm{IOS}-\mathrm{SC}}(T)\right|_{\bar{\mu}=\mu} \leq k_{10}^{\mathrm{EM}-\mathrm{SC}}(T) \leq\left. k_{10}^{\mathrm{IOS}-\mathrm{SC}}(T)\right|_{\bar{\mu}=0.9 \mu}
$$

Fig. 4 shows how the upper and lower bounds of $K_{10}^{\mathrm{EM}}$ SC embrace the experimental data points as well as the results of accurate quantum calculations. The performance of the SC method can be appreciated more when compared to CT (original LT treatment), CCT and SC calculations for a pure repulsive interaction. In particular, it shows that the main reason for the curvature of the linear CT (Landau-Teller) plot comes from the quantum effect of the liberation of large amounts of energy rather than the deviation of the repulsion from its high-energy exponential form. This conclusion may seem unexpected in view of the large deviation of the encounter time from its high-energy counterpart at, say, $100 \mathrm{~K}$ (see Fig. 3) and the rather small deviation of the accurate and EM SC rate coefficients from their SC counterpart calculated for a purely repulsive interaction. However, one notes that the SC transition probability is determined by the integral over the collision energies where the portion close to the lower limit does not play a significant role. We also observe that a similar approach reproduces well the isotope effect on collisions ${ }^{14} \mathrm{~N}_{2}+{ }^{4} \mathrm{He}$ and ${ }^{15} \mathrm{~N}_{2}+{ }^{3} \mathrm{He} .{ }^{77}$ However, along with the dependence of the SC exponent on the reduced mass of the partners and the reduced mass of the diatom, one should also take into account the dependence of the pre-exponential factor on these quantities.

\subsection{Relaxation of HF in Ar: strong effect of rotation}

The relaxation of $\mathrm{HF}$ in $\mathrm{Ar}$

$$
\mathrm{HF}(v=1)+\mathrm{Ar} \rightarrow \mathrm{HF}(v=0)+\mathrm{Ar}
$$

markedly differs from that in the $\mathrm{N}_{2}+\mathrm{He}$ system for the following reasons:

(i) The high anisotropy of the interaction, the rather large reduced mass of the partners and the small value of the moment of inertia of $\mathrm{HF}$ result in an effective mass that strongly depends on the orientation angle. This suggests that the BS and IOS models can not be applied and either the HR or the EM model have to be considered.

(ii) There exist quantum CS calculations ${ }^{107,108}$ which provide some possibility for comparison with the HR and EM results.

(iii) Unfortunately the available experimental data do not allow for an unambiguous interpretation in terms of state-tostate rate coefficients (see below). ${ }^{108}$ In this case, we refrain from comparing the theoretical and experimental values of $k_{10}$ and concentrate on a comparison of theoretical values of $k_{10}$ from the numerical CTC HR calculations of 1985 employing the best available PES at that time, $k_{10}^{\mathrm{CTC}-\mathrm{HR}}$, with numerical CS calculations of 2002 with two more recent $a b$ initio PES, $k_{10}^{\mathrm{CS}}$. We also verify qualitative predictions of the HR model about the distribution of the released energy over translational and rotational degrees of freedom.

6.2.1 Comparison of $\mathrm{HR}$ and EM results with accurate CS calculations. HR rate coefficients for HF relaxation in Ar were calculated for different potentials in ref. 75. The comparison of theoretical CCT HR rate coefficients for the PES from ref. 109 with experimental data ${ }^{110-112}$ demonstrated a fair agreement, though it was noted that the theoretical results were very sensitive to the analytical representation of the PES. The LT plots of $k_{10}^{\text {CCT-HR }}$, based on Fig. 3 from ref. 75, are presented in

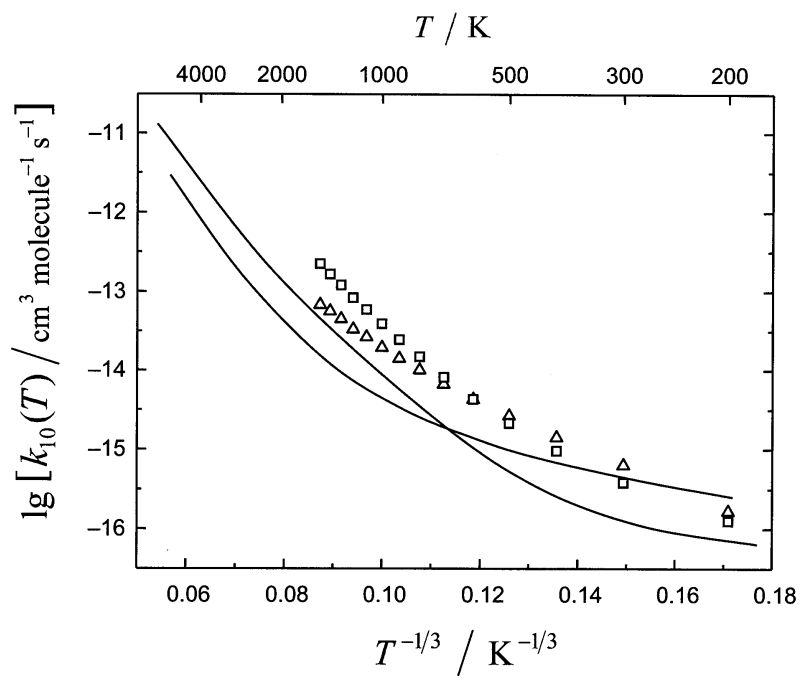

Fig. 5 Comparison of LT plots of theoretical rate coefficients for vibrational relaxation of $\mathrm{HF}$ in $\mathrm{Ar}$, for different PES calculated within the HR model and $\mathrm{CC}$ approach. The full lines correspond to $k_{10}^{\text {CTC-HR }}$ from ref. 75 for two different interpolations between the points of the same PES from ref. 109. The symbols represent $K_{10}^{\mathrm{CC}}$ from ref. 108 for two PES, see text. 
Fig. 5 by two solid lines, each corresponding to different interpolations between the data points of the same PES from ref. 109. The difference between the two lines demonstrates the sensitivity of the rate coefficient to seemingly "reasonable" modifications of the potential resulting from different global analytical fittings of the PES.

Quantum CS rate coefficients for two more recent $a b$ initio PES were reported in ref. 108. The differences between the theoretical rate coefficients and the experimental data were found to be quite large, the former being a factor of 10 or 30 higher than the latter. It was argued that neither the inaccuracy of $\mathrm{CS}$ (compared to the accurate $\mathrm{CC}$ calculations) nor reasonable variations of the PES could explain the disagreement with the experimental data. It was then concluded that the experimental data do not correspond to the rate coefficient $k_{10}$ but rather to an effective rate coefficient for a complicated sequence of events: the collision-induced near resonance transfer of vibrational energy of $\mathrm{HF}$ to its rotational energy, followed in successive collisions by the back transfer of rotation to vibration and by the collisional rotational relaxation of $\mathrm{HF}{ }^{108}$ The LT plots of $k_{10}^{\mathrm{CS}}$, based on Fig. 1 from ref. 108, in Fig. 5 are presented for two PES by symbols. The difference between the two sets of symbols demonstrates the sensitivity of the rate coefficient to seemingly "reasonable" variations of the PES.

On the whole, we can see that CCT HR rates are below the $\mathrm{CC}$ rates, the difference between the two sets of results within each approach (CCT HR or CC) is of the same order of magnitude as the difference between the results for different choices of the PES, and both lead to curvatures at lower temperatures. A convincing comparison between the HR and CS approaches should be based on calculations that use the same PES. This is not available at present. However, the CS results were compared with the IOS approximation and with a simplified version of EM model, the so called EM-IOS approximation. ${ }^{108}$ In the latter, the IOS code was supplemented with an angle-dependent effective mass suggested by eqn (5.14). The IOS model fails badly, while the EM-IOS approximation performs better, still yielding cross sections for vibrational relaxation which are about two orders of magnitude lower than the CS cross sections (Fig. 8 from ref. 107). Considering this disagreement, the authors of ref. 108 expressed the optimistic view that "by choosing the effective mass in a more appropriate way, it is possible to obtain even quantitative agreement with the more accurate CS data". We cannot exclude, however, that for relaxation of hydrogen halide molecules in collisions with heavy noble gas atoms the EM model should be abandoned and replaced by the HR model.

\subsubsection{Distribution of released energy}

As discussed in section 5, the HR model predicts that upon vibrational deactivation, the vibrational energy of $\mathrm{BC}$, at a certain distance $R$ between $\mathrm{A}$ and the center of mass of $\mathrm{BC}$, is transformed into the energy of hindered rotation of $\mathrm{BC}$. When $\mathrm{A}$ and $\mathrm{BC}$ separate, the energy of hindered rotation is partitioned into rotational energy of $\mathrm{BC}$ and relative translational energy of A-BC. This partitioning depends on the extent of how strongly, at the distance $R$, the free rotation of $\mathrm{BC}$ is perturbed by the interaction of A with $\mathrm{BC}$. For a given $j$, this perturbation becomes larger with increasing translational energy and, for a given translational energy, this perturbation diminishes with increasing $j$. These observations lead to the following qualitative conclusion about the resonance features of transformation of vibration of $\mathrm{BC}$ into free rotation of $\mathrm{BC}$ : the resonance with respect to the final values of $j=j^{\prime \prime}$ broadens with increasing initial translational energy and becomes narrower with respect to $j^{\prime \prime}$ with increasing $j^{\prime}$. The above predictions of the HR model are in agreement with the results of the CS quantum study of the relaxation of HF in Ar, as revealed from Fig. 6 of ref. 107 (distribution over $j^{\prime \prime}$ for $j^{\prime}=0$ and different collision energies $E_{t}^{\prime}=300,834,3000$, and 4000 $\mathrm{cm}^{-1}$ ) and Fig. 3 of ref. 108 (distribution over $j^{\prime \prime}$ for $E_{t}^{\prime}=2106$ $\mathrm{cm}^{-1}$ and different initial rotational quantum numbers $j^{\prime}=0$, $8,17)$. We regard this conclusion as yet another indication for the applicability of the HR model to the vibrational deactivation of $\mathrm{HF}$ in Ar.

\subsection{Relaxation of $\mathrm{HCl}$ in Ar: comparison of different driving modes}

The relaxation of $\mathrm{HCl}$ in $\mathrm{Ar}$

$$
\mathrm{HCl}(v=1)+\mathrm{Ar} \rightarrow \mathrm{HCl}(v=0)+\mathrm{Ar}
$$

exhibits features which are similar to the relaxation of $\mathrm{HF}$ in Ar. We discuss this system in order to compare the efficiency of along-the-gradient and rotational driving modes, leaving aside the BS and IOS models which for a long time were known to be inadequate for describing the relaxation of this particular system.

The most detailed study of this process within the EM approach was done in ref. 87 . The authors used the so-called "classical approach quantum encounter" method which combined the search for optimal collision configurations from classical trajectories with quantum expressions for the vibrational transition probability. The latter referred to an exponential repulsive interaction and contained the effective mass. As remarked by the authors, "this hybrid classical/quantum development may prove to be quite useful, except for very glancing collisions". The effective mass calculated for the presumably best PES (called M5), exhibits a sharp minimum at about $\gamma^{\dagger}=25^{\circ}$ with $\mu^{\dagger} \approx 7$ a.m.u. (in comparison to $\mu=19$ a.m.u.) which is reflected in a pronounced maximum of the transition probability as a function of the angle $\gamma^{*}$. The calculated values of the averaged vibrational transition probability (for the M5 PES with a well depth of $160 \mathrm{~cm}^{-1}$ ) were found to agree well with the experimental data within the temperature range 296-1953 K, when the length parameter $a$ was optimised yielding a value of $a=0.25 \AA$. When the length parameter was increased to a value of $a=0.35 \AA$ the transition probabilities drop by about two orders of magnitude (Fig. 10 from ref. 87).

A HR description of the same process was presented earlier by Ovchinnikova. ${ }^{75}$ She numerically calculated the transition probabilities from the Fourier components of the perturbation by running classical HR trajectories at fixed values of $R$ with subsequent averaging over the separations of the partners, and introducing quantum correction to the CT approach. Calculations were performed for different PES, and reasonable agreement with experimental data was found for some of these (Fig. 2 from ref. 75). The Billing potential with a well depth of 140 


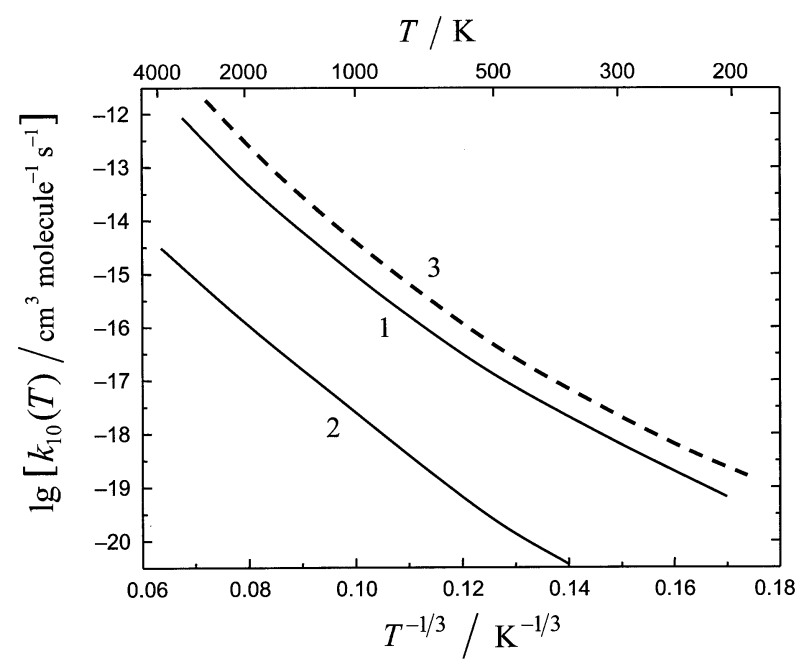

Fig. 6 LT plots of theoretical vibrational relaxation rate coefficients for $\mathrm{HCl}$ in Ar. HR and EM model calculations with the same pair-wise exponentially repulsive interaction potential, solid lines 1 and 2, respectively. The dotted line 3 corresponds to the analytical HR model with the potential from eqn (5.15) which approximates the pair-wise potential in the region of rotational encounter, see text.

$\mathrm{cm}^{-1}$ and length parameter $a=0.35 \AA{ }^{90}$ in particular, led to reasonable agreement between theoretical experimental data (Fig. 2 from ref. 75). An analysis of the contribution of rebound and glancing HR trajectories to the transition probability showed (Fig. 6 of ref. 75) that the fraction of rebound encounters for this PES was quite high (0.6-0.75) such that the hindrance of rotation is an important dynamical feature of the energy transfer.

It is difficult to discriminate between EM or HR model calculations by comparing theoretical results with experimental data, because different PES and dynamical simplifications were used in the different calculations. Also, like in HF-Ar relaxation (see above), the data may not have corresponded to state-to-state rate coefficients. A more secure insight can be gained by comparing EM and HR theoretical rate coefficients, calculated for the same PES on the same level of dynamical approximation. Fig. 6 shows results of such calculations in the CCT approximation for pair-wise exponentially repulsive interactions with the length parameter $a=0.35 \AA \AA^{96}{ }^{96}$ Curves 1 and 2 represent CCT rate coefficients for HR and EM approaches while curve 3 corresponds to the coefficients for a PES with an anisotropic term in the form of eqn (5.15).

The inspection of the graphs in Fig. 6 shows that:

(i) the temperature dependences of the rate coefficient for the initial potential and its analytical approximation are virtually the same, though the rates differ by a factor of two;

(ii) the values of $k_{10}^{\mathrm{EM}-\mathrm{CCT}}$ are much lower, and their temperature dependence is much steeper than that of $k_{10}^{\mathrm{HR}-\mathrm{CCT}}$. However, a more detailed interpretation of the difference in the temperature dependences of $k_{10}^{\mathrm{EM}-\mathrm{CCT}}$ and $k_{10}^{\mathrm{HR}-\mathrm{CCT}}$ would require the comparison not only of the exponential but also of the pre-exponential factors. This is outside the scope of this paper.

From (i) we conclude that the temperature dependence of the HR rate coefficient is not sensitive to variations in the shape of the rotational barrier (within physically reasonable limits) and, therefore, the approximation of eqn (5.15) for a strongly anisotropic interaction can be used to estimate the order-of magnitude of the HR rate coefficients. This procedure is similar to the exponential approximation in LT type BS or IOS rate coefficients in the case of interaction with weak anisotropy.

From (ii) we can see that the rotational driving mode (HR model) is by far more effective in inducing the vibrational transition than the along-the-gradient driving mode (EM model). In other words, accepting the EM model, one misses the channel which corresponds to the HR model. This conclusion still applies if one changes the value of the length parameter $a$. If the latter is forced to reproduce the experimental data within the EM model by decreasing its value from 0.35 down to 0.25 , one will still neglect the more efficient pathway of relaxation via the HR mechanism. One should have been alerted about this problem of the EM model in $\mathrm{HCl}-\mathrm{Ar}$ collisions since the angular dependence of the effective mass found in ref. 87 showed quite a narrow and pronounced minimum. The width of this minimum is quite small and the rapidly rotating molecule $\mathrm{HCl}$ can traverse it during the along-the-gradient encounter time. Under this circumstance, the effective mass approximation loses its meaning, and the EM model becomes internally inconsistent.

Since the breathing sphere (and infinite-order sudden), optimal effective mass, and hindered rotor models yield similar analytical expressions for the main temperature dependence of the rate coefficients in the case of an exponentially repulsive interaction, we finally conclude that the efficiency of different driving modes, at least at high temperatures, can by qualitatively judged on the basis of the respective LT temperatures such as they are defined in eqn (5.5) for BS, eqn (5.14) for EM and eqn (5.19) for HR models. ${ }^{113}$

\section{Conclusion}

Until the end of 1960s, the Landau-Teller model served as the generally accepted basis for the calculation of vibrational-totranslational rate coefficients and hence for the modeling of vibrationally nonequilibrium kinetic processes in gases. With the advent of new techniques for the experimental determination of vibrational state-to-state rate coefficients and the development of numerical codes for solving quantum scattering equations, the Landau-Teller model started to get out of view. Nowadays, when collisional rate coefficients can be calculated numerically with high accuracy, and master equations for nonequilibrium distribution functions can be solved rather easily, attempts to revive the Landau-Teller model as a means of the quantitative interpretation of experimental data will not be too successful and the model seems to survive only in tutorial and historical sections of reviews on collisional energy transfer. However, one should still consider the LT model as a piece of art which reflects rather than reproduces reality. Regarded in this way, generalized LT models such as those described in the present review possess qualitative predictive power and provide insight into various factors governing collisional energy transfer. One can consider these models as semiclassical counterparts of the general quantum coupled 
states approach which, in turn, represents a good approximation to the accurate close-coupling method. If one restricts this task to the determination of the temperature dependence of the vibrational relaxation rate coefficients rather than their absolute values, the semiclassical 3-D LT models in the nearadiabatic regime can serve as useful guides to the relevant molecular parameters governing energy transfer. In this sense, the Landau-Teller model even after 70 years has not lost its actuality. We finally note that, beside being practically useful, the Landau-Teller model demonstrates a fundamental relation between the classical Ehrenfest adiabatic principle, as expressed by eqn (3.2), and its semiclassical generalization, eqn (4.2).

\section{Appendix. Glossary of abbreviations used in this paper}

\begin{tabular}{ll}
\hline AO & Anharmonic oscillator \\
BS & Breathing sphere \\
BF & Body fixed \\
CC & Close coupling \\
CS & Coupled states \\
CT & Common trajectory \\
CCT & Corrected common trajectory \\
1-D & One-dimensional \\
3-D & Three-dimentional \\
EM & Effective mass \\
HO & Harmonic oscillator \\
HR & Hindered motor \\
IOS & Infinite order sudden \\
FOD & First order diabaic \\
FOA & First order adiabatic \\
LT & Landau-Teller \\
OEM & Optimal effective mass \\
PES & Potential energy surface \\
SC & Semiclassical \\
SD & Steepest descent \\
SOD & Second order diabatic \\
SSH & Schwartz-Slawsky-Herzfeld \\
VT & Vibration-translation \\
VP & Vibrational predissociation \\
WKB & Wentzel-Kramers-Brillouin \\
& \\
\hline &
\end{tabular}

\section{Acknowledgements}

In writing this article, the authors benefited to a large extent from scientific contacts with their colleagues and co-workers. In particular, E.E.N. enjoyed years-long fruitful collaboration with E. I. Dashevskaya, M. Ya. Ovchinnikova, D. V. Shalashilin, S. Ya. Umanskii at the Institute of Chemical Physics, Moscow, and with Y. Karni and I. Litvin at the Schulich Faculty of Chemistry, Technion, Haifa. E.E.N. and J.T. acknowledge enlightening discussions with D. Schwarzer, J. Schroeder, G. Käb and A. A. Neufeld at the MPI für Biophysikalische Chemie, Göttingen.

\section{References}

1 L. Landau and E. Teller, Phys. Z. Sow., 1936, 10, 34.

2 A. Eucken and H. Jaacks, Z. Phys. Chem., 1935, 30, 85.

$3 \mathrm{H}$. Bethe and E. Teller, Deviations from thermal equilibrium in shock waves, Ballistic Research Laboratory, Aberdeen proving ground, Report X-117, 1941.

4 R. N. Schwartz, Z. I. Slawsky and K. F. Herzfeld, J. Chem. Phys., 1952, 20, 1591.

5 J. M. Jackson and N. F. Mott, Proc. R. Soc. London, Ser. A, 1932, 137, 703.

6 K. Takayanagi, Progr. Theor. Phys., 1952, 8, 497.

7 R. N. Schwartz and K. F. Herzfeld, J. Chem. Phys., 1954, 22, 767.

8 T. L. Cottrell and J. C. McCoubrey, Molecular Energy Transfer in Gases, Butterworths, London, 1961.

9 E. E. Nikitin, Theory of Elementary Atomic and Molecular Processes in Gases, Clarendon Press, Oxford, 1974.

10 Dynamics of Molecular Collisions, Parts A and B, ed. W. H. Miller, Plenum Press, New York, 1976.

11 Atom-Molecule Collision Theory: a Guide for the Experimentalists, ed. R. B. Bernstein, Plenum Press, New York, 1979.

12 F. A. Gianturco, The Transfer of Molecular Energies by Collision, Springer, Berlin, 1979.

13 D. C. Clary, in Supercomputer Algorithms for Reactivity, Dynamics and Kinetics of Small Molecules, ed. A. Lagana, NATO ASI, Kluwer, Dordrecht, 1989.

14 L. Landau, Phys. Z. Sow., 1932, 2, 46.

15 Y. Karni and E. E. Nikitin, J. Chem. Phys., 1994, 100, 2027.

16 E. E. Nikitin and L. Pitaevski, Phys. Rev. A: At., Mol., Opt. Phys., 1994, 49, 695.

17 J. H. Jeans, Philos. Mag., 1903, 6, 279.

18 C. Zener, Phys. Rev., 1931, 38, 277.

19 J. E. Lennard-Jones and C. Strachan, Proc. R. Soc. London, Ser. $A, 1935,150,442$.

20 C. Strachan, Proc. R. Soc. London, Ser. A, 1935, 150, 456.

21 J. E. Lennard-Jones and A. F. Devonshire, Proc. R. Soc. London, Ser. A, 1936, 156, 6.

22 L. Landau and E. Lifshitz, Classical Mechanics, Pergamon Press, Oxford, 1970.

23 L. Landau and E. Lifshitz, Quantum Mechanics (Non-relativistic Theory), Pergamon Press, Oxford, 1977.

24 M. Baer, G. Drolshagen and J. P. Toennies, J. Chem. Phys., 1980, 73, 1690 .

25 G. Drolshagen, J. P. Toennies and M. Baer, Chem. Phys. Lett., 1983, 102, 354.

26 E. P. Gordeev and S. Ya. Umanskii, Khim. Fiz., 1984, 3, 649.

27 Y. Karni and E. E. Nikitin, Chem. Phys., 1995, 191, 235.

28 Y. Karni and E. E. Nikitin, Mol. Phys., 1996, 89, 1327.

29 R. J. Rubin and K. E. Shuler, J. Chem. Phys., 1956, 25, 59.

30 E. W. Montroll and K. E. Shuler, J. Chem. Phys., 1957, 26, 452.

31 K. E. Shuler, Phys. Fluids, 1959, 2, 442.

32 Nonequlibrium Vibrational Kinetics, ed. M. Capitelli, Springer, Berlin, 1986.

33 S. W. Benson, G. C. Berend and J. C. Wu, J. Chem. Phys., 1963, 38, 25.

34 S. J. Yao and H. Y. S. Yao, J. Chem. Phys., 1971, 54, 4424

35 H. A. Kramers, Physica, 1940, 7, 284.

36 D. W. Oxtoby, Adv. Chem. Phys., 1981, 47, 487.

37 S. A. Egorov and J. L. Skinner, Chem. Phys. Lett., 1998, 283, 469.

38 S. A. Egorov, E. Rabinim and B. J. Berne, J. Phys. Chem. B, 1999, 103, 10978.

39 G. Käb, Phys. Rev. E, 2002, 66, 046117.

40 G. Käb, J. Phys. Chem. A, 2004, 108, 8866.

41 A. A. Neufeld, J. Chem. Phys., 2003, 119, 2488.

42 A. A. Neufeld, D. Schwarzer, J. Schröder and J. Troe, J. Chem. Phys., 2003, 119, 2502.

43 Q. Shi and E. Geva, J. Phys. Chem. A, 2003, 107, 9059.

44 Q. Shi and E. Geva, J. Phys. Chem. A, 2003, 107, 9070.

45 B. Widom, Discuss. Faraday Soc., 1962, 33, 37.

46 A. F. Devonshire, Proc. R. Soc., 1937, 21, 269.

47 A. Miklave, J. Chem. Phys., 1983, 78, 4502.

48 E. E. Nikitin, Opt. Spektr., 1959, 6, 93.

49 P. Schofield, Phys. Rev. Lett., 1960, 4, 239.

50 E. E. Nikitin and J. Troe, Phys. Chem. Chem. Phys., 2006, 8, 2012. 
51 D. Schwarzer and M. Teubner, J. Chem. Phys., 2002, 116, 5680. 52 M. Teubner, Phys. Rev. E, 2002, 65, 031204.

53 G. D. Billing, The Quantum Classical Theory, University Press, Oxford, 2003.

54 E. W. Montroll, R. J. Rubin and K. E. Shuler, J. Chem. Phys., 1958, 28, 700.

55 E. E. Nikitin, Doklady AN SSSR, 1959, 124, 1085.

56 J. A. Beswick and J. Jortner, J. Chem. Phys., 1978, 68, 2277.

57 J. A. Beswick and J. Jortner, J. Chem. Phys., 1978, 69, 512.

58 J. A. Beswick and J. Jortner, Adv. Chem. Phys., 1981, 47, 363.

59 M. S. Child, Semiclassical Mechanics with Molecular Applications, Clarendon Press, Oxford, 1991.

60 G. E. Eving, J. Chem. Phys., 1979, 71, 3143.

61 M. Willberg, M. Gutmann, E. E. Nikitin and A. H. Zewail, Chem. Phys. Lett., 1993, 201, 506.

62 S. B. Woodruff and D. L. Thompson, J. Chem. Phys., 1979, 71, 376.

63 S. K. Gray, S. A. Rice and D. W. Noid, J. Chem. Phys., 1986, 84, 3745 .

64 D. W. Noid, S. K. Gray and S. A. Rice, J. Chem. Phys., 1986, 84, 2649.

65 M. J. Davis and S. K. Gray, J. Chem. Phys., 1986, 84, 5389.

66 S. K. Gray, S. A. Rice and M. J. Davis, J. Phys. Chem., 1986, 90, 3470 .

67 K. Gray, J. Chem. Phys., 1987, 87, 2051.

68 B. V. Chirikov, Phys. Rep., 1979, 52, 265.

69 M. G. Gutzwiller, Chaos in Classical and Quantum Mechanics, Springer, New York, 1990.

70 E. I. Dashevskaya, I. Litvin, E. E. Nikitin, I. Oref and J. Troe, Phys. Chem. Chem. Phys., 2000, 2, 225.

71 E. I. Dashevskaya, I. Litvin, E. E. Nikitin and J. Troe, Phys. Chem. Chem. Phys., 2001, 3, 2315.

72 E. I. Dashevskaya, I. Litvin, E. E. Nikitin, I. Oref and J. Troe, Phys. Chem. Chem. Phys., 2002, 4, 3330.

73 E. E. Nikitin and J. Troe, in Theory of Chemical Reaction Dynamics, ed. A. Lagana and G. Lendvay, Kluwer, Dordrecht, 2004.

74 Y. Karni and E. E. Nikitin, J. Chem. Phys., 1994, 100, 8065.

75 M. Ya. Ovchinnikova, Chem. Phys., 1985, 93, 101.

76 E. E. Nikitin, A. I. Osipov and S. Ya. Umanskii, in Khimiya Plazmy, Energoatomizdat, Moscow, 1989.

77 E. I. Dashevskaya, I. Litvin, E. E. Nikitin and J. Troe, J. Chem. Phys., 2006, 125, 154315.

78 E. E. Nikitin, Teor. Eksper. Khim., 1967, 3, 185.

79 G. A. Kapralova, E. E. Nikitin and A. M. Chaikin, Chem. Phys. Lett., 1968, 2, 581.

80 C. B. Moore, J. Chem. Phys., 1965, 43, 2979.

81 A. Miklave, Mol. Phys., 1980, 39, 855.

82 A. Miklave, J. Chem. Phys., 1980, 72, 3805.

83 A. Miklave, J. Chem. Phys., 1983, 78, 4502.

84 B. D. Cannon and I. W. M. Smith, Chem. Phys., 1984, 83, 429.
85 A. Miklavc and I. W. M. Smith, J. Chem. Soc., Faraday Trans. 2, $1988, \mathbf{8 4}, 227$.

86 A. Miklavc, N. Marković, G. Nyman, V. Harb and S. Nordholm, J. Chem. Phys., 1992, 97, 3348.

87 T. D. Sewell, S. Nordholm and A. Miklavc, J. Chem. Phys., 1993, 99, 2567.

88 E. E. Nikitin, Khim. Fiz., 1993, 12, 187.

89 F. A. Gianturco, J. P. Toennies and M. Bernardi, J. Chem. Soc., Faraday Trans., 1991, 87, 31.

90 G. D. Billing, J. Chem. Phys., 1972, 57, 5241.

91 H. K. Shin and A. W. Young, J. Chem. Phys., 1974, 60, 193.

92 I. V. Lebed' and S. Ya. Umanski, Teor. Eksp. Khim., 1978, 14, 102.

93 M. Ya. Ovchinnikova and D. V. Shalashilin, Khim. Fiz., 1984, 3, 380.

94 M. Ya. Ovchinnikova and D. V. Shalashilin, Khim. Fiz., 1984, 3, 643.

95 M. Ya. Ovchinnikova, Khim. Fiz., 1985, 4, 867.

96 E. E. Nikitin and M. Ya. Ovchinnikova, Khim. Fiz., 1986, 5, 291.

97 E. E. Nikitin, Khim. Fiz., 1986, 5, 1155.

98 E. E. Nikitin and D. V. Shalashilin, Khim. Fiz., 1992, 11, 1471.

99 E. E. Nikitin, S. Ya. Umanski and D. V. Shalashilin, Khim. Fiz., $1989,8,1011$.

100 J. P. Reid, A. J. Thakkar, P. W. Barnes, E. F. Archibong, H. M. Quiney and C. J. S. M. Simpson, J. Chem. Phys., 1997, 107, 2329.

101 J. P. Reid, C. J. S. M. Simpson and M. Quiney, Chem. Phys. Lett., 1996, 256, 531.

102 D. R. White, J. Chem. Phys., 1968, 48, 525.

103 M. M. Maricq, E. A. Gregory, C. T. Wickham-Jones, D. J. Cartwright and C. J. S. M. Simpson, Chem. Phys., 1983, $\mathbf{7 5}, 347$.

104 A. Banks, D. C. Clary and H. J. Werner, J. Phys. Chem., 1986, 84, 3788.

105 E. E. Nikitin and S. Ya. Umanskii, Theory of Slow Atomic Collisions, Springer, Berlin, 1984.

106 K. T. Tang, J. P. Toennies and C. L. Yiu, Int. Rev. Phys. Chem., 1998, 17, 363.

107 R. V. Krems, N. Marković, A. A. Buchachenko and S. Nordholm, J. Chem. Phys., 2001, 114, 1249.

108 R. V. Krems, A. A. Buchachenko, N. Marković and S. Nordholm, J. Chem. Phys., 2002, 117, 166.

109 T. F. Eving, J. Detrich and R. W. Conne, J. Chem. Phys., 1978, 69, 4662.

110 J. F. Bott and N. J. Cohen, J. Chem. Phys., 1971, 55, 368.

111 G. K. Vasiliev, E. F. Makarov, V. G. Papin and V. L. Tal'rose, Zh. Eksp. Teor. Fiz., 1973, 64, 2946.

112 L. S. Blair, W. D. Breshears and G. L. Schoff, J. Chem. Phys., 1973, 59, 1582.

113 E. E. Nikitin, in Gas Phase Chemical Reaction System, ed. J. Wolfrum, H.-R. Volpp, R. Rannacher and J. Warnatz, Springer, Berlin-Heidelberg, 1996. 